\title{
Modelling and Monitoring Erosion of the Leading Edge of Wind Turbine Blades
}

\author{
Gregory Duthé ${ }^{1, \ddagger *(\mathbb{D})}$, Imad Abdallah ${ }^{1, \ddagger(1)}$, Sarah Barber ${ }^{2}$, and Eleni Chatzi ${ }^{1}(\mathbb{D})$ \\ 1 Institute of Structural Engineering, ETH Zürich, Switzerland \\ 2 Eastern Switzerland University of Applied Sciences, Switzerland \\ * Correspondence: duthe@ibk.baug.ethz.ch \\ $\ddagger \quad$ These authors contributed equally to this work.
}

Citation: Duthé, G.; Abdallah, I.; Barber, S; Chatzi, E. Title. Journal Not Specified 2021, 1, 0. https://doi.org/

Received:

Accepted:

Published:

Publisher's Note: MDPI stays neutral with regard to jurisdictional claims in published maps and institutional affiliations.

Copyright: () 2021 by the authors. Submitted to Journal Not Specified for possible open access publication under the terms and conditions of the Creative Commons Attribution (CC BY) license (https:// creativecommons.org/licenses/by/ $4.0 /)$.

\begin{abstract}
Leading edge surface erosion is an emerging issue in wind turbine blade reliability, causing reduction in power performance, aerodynamic loads imbalance, increased noise emission and ultimately additional maintenance costs, and if left untreated, leads to the compromise of the functionality of the blade. In this work, we first propose an empirical spatio-temporal stochastic model for simulating leading edge erosion, to be used in conjunction with aeroelastic simulations, and subsequently propose a deep learning model trained on simulated data, which aims to monitor leading edge erosion by detecting and classifying the degradation severity. The main ingredients of the model include a damage process that progresses at random times, across multiple discrete states characterized by a non-homogeneous compound Poisson process, which is used to describe the random and time-dependent degradation of the blade surface, thus implicitly affecting its aerodynamic properties. The model allows for one, or more, zones along the span of the blades to be independently affected by erosion. The proposed model accounts for uncertainties in the local airfoil aerodynamics via parameterization of the lift and drag coefficients curves. The proposed model is used to generate a stochastic ensemble of degrading airfoil aerodynamic polars, for use in forward aero-servo-elastic simulations, where we compute the effect of leading edge erosion degradation on the dynamic response of a wind turbine under varying turbulent input inflow conditions. The dynamic response is chosen a defining output as this relates to the output variable that is most commonly monitored under a Structural Health Monitoring (SHM) regime. In this context, we further propose an approach for spatio-temporal dependent diagnostics of leading erosion, namely, a deep learning attention-based Transformer, which we modify for classification tasks on slow degradation processes with long sequence multivariate time-series as inputs. We perform multiple sets of numerical experiments, aiming to evaluate the Transformer for diagnostics and assess its limitations. The results reveal Transformers as a potent method for diagnosis of such degradation processes. The attention-based mechanism allows the network to focus on different features at different time intervals for better prediction accuracy, especially for long time-series sequences representing a slow degradation process.
\end{abstract}

27 Keywords: Wind turbine; Structural Monitoring; Leading edge erosion; Poisson process; aeroelas28 tic simulations; Lift and drag; Deep learning; Transformer; Diagnostics 


\section{${ }_{29}$ Nomenclature}

sо $\quad \alpha \quad$ Wind shear exponent

${ }_{31} \quad \mathbb{E} \quad$ Expected value of a random variable

32 $\mathbb{V} \quad$ Variance of a random variable

$33 \quad \sigma \quad$ Turbulence

${ }_{34} \quad C_{D} \quad$ Aerodynamic drag coefficient

${ }_{35} C_{L} \quad$ Aerodynamic lift coefficient

${ }_{36} C_{m} \quad$ Aerodynamic moment coefficient

${ }_{37} C_{p} \quad$ Aerodynamic pressure coefficient

${ }_{38}$ CNN Convolutional Neural Network

39 EZ Eroding Zone

$40 \quad L E$ Leading Edge

${ }_{41}$ LEE Leading Edge Erosion

42 MLP Multilayer Perceptron

${ }_{43}$ MTS Multivariate time-series

${ }_{44}$ NHCPP Non-Homogeneous Compound Poisson Process

45 O\&M Operation and Maintenance

${ }_{46} \quad R \quad$ Blade radius

47 RNN Recurrent Neural Network

${ }_{48} \quad R V \quad$ Random Variable

49 SHM Structural Health Monitoring

so $\quad T_{i} \quad$ Turbulence intensity

${ }_{51} \quad U \quad$ Mean wind speed

${ }_{52}$ WT Wind Turbine

\section{${ }_{53} 1$ Introduction}

This work is motivated by current efforts on development of a novel micro-electromechanical monitoring system based on aerodynamic surface pressure and aero-acoustic measurements for Structural Health Monitoring of wind turbine blades, as part of the Aerosense project ${ }^{1}$. Sensing nodes are distributed along the span of the blade delivering measurements of local sectional aerodynamic pressure distributions, aeroacoustic and acceleration signals (see illustration in Figure 1). Assuming availability of such a monitoring system, we here propose an approach to the modelling and diagnostics of erosion of the leading edge for wind turbine blades. 


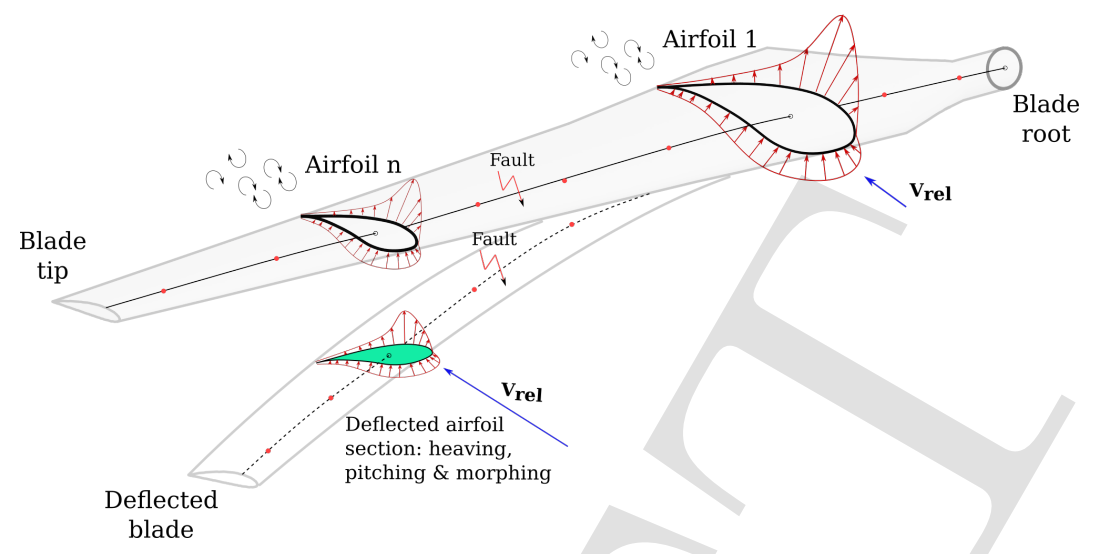

Figure 1. Illustration of Aerosense, a novel MEMS-based aerodynamic surface pressure and aero-acoustic measurement system for wind turbines blades.

Bogdanoff and Kozin (1985) [1] define cumulative damage as the "irreversible accumulation of damage throughout life that ultimately leads to failure". Leading edge erosion of a wind turbine blade represents such a process, albeit at slow degradation rates, with effects slow to manifest and even harder to detect early on. Most diagnostic techniques today rely on direct visual inspection[2], image processing [3], and statistical analysis, e.g. regression or clustering of SCADA output, such as blade pitch, rotor speed or electrical power [4,5]. Visual inspection and image acquisition imply stopping the turbines, resulting in loss of power production. More importantly, they give a local (both spatial and temporal) snapshot of the erosion condition without a direct link to the historical progression of the degradation and how it relates to environmental and operational conditions. Even though it is better positioned to discriminate the environmental and operational conditions versus degradation process, long term SCADA based statistical analysis invariably do not possess the necessary resolution to determine accurately the severity and spatial extent of erosion on the blades. A gap therefore exists in the field of spatio-temporal modelling and diagnostics of leading edge erosion for wind turbines rotor blades. In this work, we are particularly interested in leveraging time series emanating from the novel Aerosense based aerodynamic surface pressure monitoring system for wind turbines blades. The aim is to (1) detect the occurrence of erosion and (2) classify the severity (intensity) of erosion. This could be regarded as either the inverse problem of reconstruction for the lift and drag coefficients, or a problem of finding the optimal discriminants for erosion versus other effects from time series response signals. This is a time dependent multi-class and multi-label problem. To tackle this problem and build meaningful diagnostics models, realistic time-series data is required for training. Our options are either collect multi-year field data, where the rotor blade are known to undergo leading erosion, or develop a numerical model of leading edge erosion coupled to an aeroelastic simulator. As data availability for such systems is often limited and restricted by data sharing protocols, we here opt for the route of simulation, with the additional benefit of delivering a model that can be used for simulating such slow leading edge erosion degradation processes.

Towards accomplishing the goal of diagnostics and inference of erosion of the leading edge of wind turbine blades, this work makes the following contributions:

- We develop a stochastic spatio-temporal erosion model of the leading edge of wind turbine blades, which is characterized by a non-homogeneous compound Poisson process across discrete states, embedded in a generator of stochastic ensemble of degrading airfoil aerodynamic polars for use in forward aero-servo-elastic simulations. The coupled model is able to compute the aeroelastic non-stationary response of a wind turbine, thus reflecting its behaviour under the effect of leading edge erosion 
A graphical abstract of this research is presented in Figure 2.

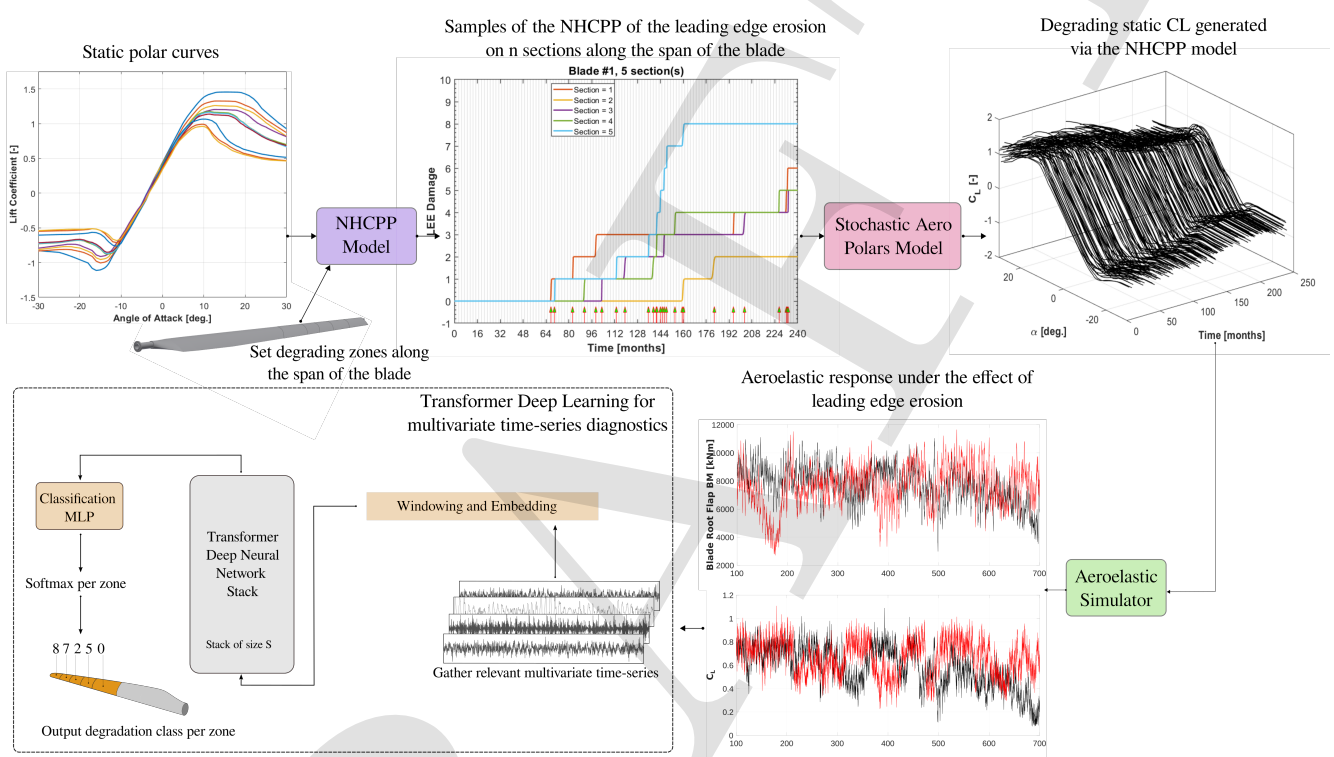

Figure 2. A graphical abstract of modelling and diagnosing erosion of the leading edge of wind turbine blades.

The remainder of this article is organized as follows. In Section 2 we offer a review of the state of the art in modelling and diagnostics of leading edge erosion. In Section 3 we present the details of the spatio-temporal stochastic model for leading edge erosion. In section 4 we present the uncertainty modelling and the aeroelastic simulations setup. In section 5 we elaborate the theory of the deep learning multi-variate time-series based Transformer for diagnostics and inference. In Section 6, we illustrate the novelty and principles of the proposed framework on a simulations-based application and further provide discussions and list the limitations in Section 7.

\section{Review of Prior Art}

We first review modelling approaches of leading edge erosion in conjunction with aeroelastic simulations and subsequently assess the state-of-the-art in diagnostics methods.

\subsection{Modelling leading edge erosion}

Several studies have quantified the impact of leading edge erosion on aerodynamic and aeroelastic performance of wind turbines. 
The general thread in the first set of studies amounts to describing the constitutive laws, physical processes and instigators at the micro scale resulting in surface erosion/degradation and the various stages and types of erosion severity e.g., [6-10]. Such models incorporate the effect of wind speed, air density, particle size, incubation time, and erosion intensity due to rainfall, snowfall, sea spray, and fog. It is unclear however how the micro-scale models couple to the macroscale aspects of leading edge erosion and consequently the effect on turbine performance.

The second set of studies quantifies the impact on wind turbine performance via computational approaches (aeroelastic or CFD simulations) or extensive experimental campaigns in wind tunnels, coupled to uncertainty propagation schemes e.g., [11-16]. The limitations in all these works is that they do not allow for multiple zones along the span of the blades to be independently affected by stochastic erosion processes.

Bortolotti et al. [17] proposes a simple stochastic model to describe the extent of blade span-wise degradation due to leading edge erosion via a single factor that is assumed to follow a truncated beta distribution. However, the long term temporal degradation process of blade leading edge is not accounted for. Dimitrov [18] describes a first attempt to introduce temporal progression to the damage growth for leading edge erosion due to rainfall events indirectly via loss of annual energy production.

To the best of our knowledge, we find a gap in existing literature for comprehensive models that encompass both stochastic spatial and temporal elements to describe the erosion process of the leading edge of wind turbine blades and the effects on their performance over long periods of degradation time horizons.

\subsection{Diagnosing leading edge erosion}

We here review state-of-the-art in leading edge erosion diagnostics and discuss potential approaches from recent work in probabilistic and machine learning research to solve the problem of spatio-temporal dependent diagnostics. The review is organized along two axes: (1) whether or not a forward model is known and to what extent it is used in training and testing and (2) whether or not the learning is supervised or unsupervised, i.e., whether or not the training relies on access to matched ground truth and measurement pairs.

Different approaches exist to diagnosing the extent of leading edge erosion on wind turbine blades. Traditionally, manual human inspection is used, although modern visual approaches are being developed to utilize drones paired with computer vision [19]. Visual inspection, however, requires the turbine to be shutdown and is highly dependent on weather conditions. Our aim is to use data-driven continuous monitoring methods to perform diagnostics without resorting to turbine shutdown. We review different machine learning and probabilistic approaches that have been put forth and which, given data recorded by sensors placed on the wind turbine (e.g. novel aerodynamic pressure sensors, SCADA, accelerometers, strain gauges, etc.), would be able to estimate the extent of leading edge erosion on wind turbine blades. In this context, the diagnostics task can be described as a multivariate time-series (MTS) classification/clustering problem; an algorithm is tasked with predicting the degradation status/class of a blade by analysing multiple time-dependent signals. We therefore include MTS methods, which have not been specifically applied to wind turbine blades in the past, allowing us to explore promising machine learning approaches from diverse fields unrelated to structural health monitoring: activity recognition, finance, anomaly detection, sound (speech, music) analysis, seismology, meteorology, human health signal (EEG and ECG) interpretation, etc. If the forward model of the degradation process is known, the diagnostics problem 
becomes a supervised classification task, seeing that each MTS data point is associated with its degradation ground truth. Data-driven MTS classification is a field which is currently generating a lot of attention, with many promising methods that have been recently developed [20]. Within these approaches, we can differentiate between those that use the raw time-series data [21], known as end-to-end approaches, and those which first extract features that are then passed to a standard classification algorithm [22].

Extracting features from time-series data can be accomplished in many ways, with domain dependant feature engineering or statistical methods often being the first choices. Algorithms based on Empirical Mode Decomposition (EMD) are implemented for representing MTS as a superposition of simpler well-behaved components called Intrinsic Mode Functions (IMFs). EMD approaches are suitable for damage detection and degradation state recognition in the analysis of nonlinear and non-stationary signals. However, they have shown low performance in highly-stochastic signals with spike pulses and jumps $[23,24]$. Distance-based metrics, which evaluate the similarity between timeseries data form another popular class of conventional feature construction methods [25]. Alternatively, bag-of-pattern models [26] [27] can also be used to generate features from sequential inputs. These methods work by dividing the time-series sample into subsequences that are then turned into an ensemble of discrete identifiers and finally a word count histogram, performed on the ensemble, is used to generate a feature vector. In [28] and [29], feature vectors are built from MTS data by passing the inputs through numerous random convolutional kernels. This approach, named ROCKET, has a relatively low computational cost, yet, when combined with a standard ridge classifier, it achieves state-of-the-art accuracy on the UCR time-series archive dataset [30]. Other methods turn the time-series inputs into feature images [31], which are then fed into Convolutional Neural Networks (CNNs) [32] designed for image classification, thus taking advantage of the recent advances in computer vision performance. Although feature engineering and feature extraction can potentially lead to accurate diagnostics with good explainability, we are interested in implementing end-to-end methods that use raw MTS data. This is due to the fact that feature-based methods often require large amounts of cumbersome human intervention, which limits generalization. Robustness is another potential downside, where changing the input, due to sensor malfunction for instance, might require re-selecting relevant features, while it can be factored into end-to-end approaches, provided it is part of the training data.

Conventional ('shallow') data-driven classification methods do not often use untransformed MTS as inputs, due to the difficulty associated with extracting relevant information from noisy, complex data. In contrast, deep learning methods often excel at learning hidden discriminative features, making them particularly well suited for end-to-end approaches [33]. CNNs have been applied to end-to-end MTS classification in the context of prognostic health management [34], surgeon skill classification [35] and other tasks [36]. CNNs are limited by their receptive field, making their use limited to relatively short time-series. Recurrent neural networks (RNNs) [37] are, by design, effective at solving tasks relating to sequential data. Long Short Term Memory (LSTM) Networks [38], currently the most commonly used type of RNNs, have been used to successfully classify clinical measurements [39] and action recognition tasks [40]. However, RNNs are also limited to relatively short input sequences due to memory requirements and are difficult to train. In recent years, Transformers [41] have attracted a lot of attention in the natural language processing community and have mostly replaced RNNs. These neural networks leverage the power of attention mechanisms to learn the relevant interdependencies within sequences. Transformers have increasingly been applied in other domains, such as computer vision [42], time-series forecasting [43], and multivariate time-series classification [44]. Graph Neural Networks [45], a class of deep data-driven methods which utilizes graph-structured data, have also been applied to end-to-end MTS classification [46]. This is done by first constructing an adjacency matrix 
from the untransformed MTS data. In [47], spatio-temporal Graph Transformer Neural Networks (GTNN) are implemented in order to effectively capture both dynamic spatial and temporal trends. We could envisage using a GTNN model to leverage temporal and spatial correlations in the degrading sections of the three blades on a wind turbine.

As previously mentioned, deep end-to-end models are in general better suited for generalisation than feature-extraction approaches. Moreover, they can be further optimised for transferability [48], for instance by applying transfer learning methods, such as using domain adaption layers [49].

When the forward model of the degradation process is unknown, other methods for ground truth gathering must be considered, such as hand-labeling individual samples; a process which is often prohibitively time-consuming for large datasets. Instead, assuming that the labels are unknown, one can use unsupervised MTS clustering machine learning methods. In this type of approach, the data-driven algorithm learns to automatically cluster MTS samples into groups that exhibit similar degradation states [50]. Most MTS clustering methods are based on feature-extraction. However, it can be noted that in an unsupervised learning context, feature-extraction has a considerable impact on clustering performance [51], and it is therefore difficult to rely on standard domain-agnostic methods to construct features. In response to this difficulty, an emerging trend has been to learn the optimal feature representation of MTS data for clustering using neural networks [52], [44].

It is also worth mentioning unsupervised MTS anomaly detection methods [53], as these binary clustering approaches could be potentially extended to diagnostics. Anomaly detection for MTS is often based on similarity metrics [26,54,55], although these approaches are limited when it comes to capturing non-linearity and contextual anomalies (i.e. behavior that is abnormal only given the time at which it occurs). Neural networks have proven to be effective at detecting non-linear anomalies [56]. LSTMs were used to detect web traffic anomalies in [57], and [58] proposed the use of CNNs to detect abnormal behavior at multiple scales. General Adversarial Networks [59], a learning framework where two neural networks compete in a zero-sum game, have also been applied to anomaly detection [60]. Contrastive learning [61] is another approach to unsupervised clustering from multivariate time series signals for fault detection and diagnostics in the context of changing operating conditions and novel fault types. Franceschi et al. [52] showed the scalability of contrastive learning via triplet losses on long time series without labeling and without structural assumptions on non-temporal data. It is expected however that the performance of contrastive learning to suffer under highly stochastic input and operating conditions as is the case in wind turbines applications.

In the context of data-driven wind turbine blade condition monitoring, a number of methods have been reported in the literature [62]. In [63] vibrational frequency response functions were used as input features to neural networks, in order to detect changes in the structural health of a blade. LSTMs were trained to classify faults, based on multivariate sensor recordings of a model wind turbine test rig in [64], while [65] implemented a CNN to detect possible wind turbine blade breakages based on SCADA data. In [66], Support Vector Machines were trained on features built from active acoustic recordings, to diagnose healthy and damaged states of a model wind turbine. Another featureextraction approach was combined with decision trees in [67] to diagnose different fault scenarios for model wind turbine. Finally, ice buildup on wind turbine blades was predicted with random forests trained on SCADA data in [68], while Weijtjens et al. [69] used Gaussian Processes trained on data emanating from low-cost tower-mounted sensors to do so. 
Considering their extensive use in other domains, Transformers bear great potential for SHM damage detection. Some researchers have made use of attention mechanisms similar to those found in Transformers for bearing remaining useful life prediction [70], but to the best of the authors' knowledge, only in [71] have proper Transformer models been used in this context. In this work, however, an end-to-end approach is not used; instead, the model is fed a pre-processed input composed of time series features such as Mel Frequency Cepstral Coefficients (MFCCs) and Short Time Fourier Transformation. We choose Transformers as the base of our end-to-end approach for diagnostics. However, we implement additional modifications so as to be able to handle multivariate sequences on the order of several 10's of thousands of data points while still being able to track long term degradation progression, reflected by newly sampled sequences with possible discontinuities. Few, if any, of the existing MTS learning models discussed above are capable of this.

\section{Modelling Erosion of the Leading Edge}

\subsection{Non-Homogeneous Compound Poisson Process}

Leading edge erosion (LEE) is caused by environmental variations in the blade surface temperature oscillations, moisture, UV radiation, raindrops, sand, hailstones or other particles impacting the leading edge of the blade. It could also be initiated by surface cracks due to global strain from blade flexing [8], errors during manufacturing in terms of paint deposits, consistency of the gel coat thickness and bonding strength, or initiating surface damage during blade handling in transport or installation. This causes the surface material to be removed from the blade surface, leaving a rough profile that degrades the aerodynamic performance and, on the long term and if left untreated, impacts the structural integrity of the blade. Blade erosion may be more predominant in the sectional regions, where the local flow velocity is higher as this induces more energetic impacts with soiling or eroding elements. This is further exacerbated by the relatively greater impact the outboard region has on overall rotor performance. In addition, the Reynolds number and inflow turbulence influence the airfoil transition location and subsequently the aerodynamic performance of the wind turbine blades. As a result, it is not plausible to simulate all possible stages of LE erosion and uncertain inflow conditions; we therefore opt for a more pragmatic approach. Erosion initially initiates on the blades in the form of pits near the leading edge on the pressure side. These pits develop gradually over time into gouges, then steadily grow in their size and density to coalesce as delaminations. Gaudern [7] identifies five categories of severity based on proprietary inspection reports and photographs of Vestas turbines that have been operating for up to 5 years. In Sareen et al. [6] we find an analysis of the effect of erosion at the leading edge on the aerodynamic performance of the DU96 - W - 180 airfoil, and categorized erosion from pits to delamination in different types and stages, as shown in Table 1. Each combination of type and stage of erosion has associated with it aerodynamic polar curves $\left(C_{L}, C_{D}, C_{M}\right)$, derived via measurements in a wind tunnel $[6,7,11]$. Throughout the paper we adopt the labeling of the leading edge erosion severity shown in Table 2.

We propose a two-step empirical spatio-temporal stochastic model of LEE. Step 1 of the model includes a damage process that occurs at random times emulating the nonstationary time of arrival of degradation and its magnitude, with 10 discrete states (what we call erosion severity as shown in Table 2) based on Non-Homogeneous Compound Poisson Process (NHCPP) to describe the random degradation of the blade surface and implicitly its aerodynamic properties [6]. In step 2 of the model, the local leading edge degradation manifests by physical changes in the aerodynamic polars. This step 
Table 1: Types and stages of leading edge erosion with the number of pits $(\mathrm{P})$, number of gouges $(G)$, and magnitude of leading edge delamination (DL) [6].

\begin{tabular}{llll}
\hline & Type A & Type B & Type C \\
\hline Stage 1 & $100 \mathrm{P}$ & - & - \\
Stage 2 & $200 \mathrm{P}$ & 200P/100G & - \\
Stage 3 & $400 \mathrm{P}$ & $400 \mathrm{P} / 200 \mathrm{G}$ & $400 \mathrm{P} / 200 \mathrm{G} / \mathrm{DL}$ \\
Stage 4 & - & $800 \mathrm{P} / 400 \mathrm{G}$ & $800 \mathrm{P} / 400 \mathrm{G} / \mathrm{DL}+$ \\
Stage 5 & - & - & $1600 \mathrm{P} / 800 \mathrm{G} / \mathrm{DL}++$ \\
\hline
\end{tabular}

Table 2: Class labels adopted in this research for the severity categorization of leading edge erosion.

\begin{tabular}{ll}
\hline Severity class & Type \& stage \\
\hline 0 & Clean airfoil \\
1 & Type A, Stage 1 \\
2 & Type A, Stage 2 \\
3 & Type A, Stage 3 \\
4 & Type B, Stage 2 \\
5 & Type B, Stage 3 \\
6 & Type B, Stage 4 \\
7 & Type C, Stage 3 \\
8 & Type C, Stage 4 \\
9 & Type C, Stage 5 \\
\hline
\end{tabular}

generates an ensemble of stochastic aerodynamic polars for a given erosion severity of LEE in NHCPP by parameterizing the lift and drag coefficients curves [72].

The stochastic degradation process of a wind turbine blade LEE is given by a non-homogeneous Poisson process (NHCPP) over a finite time horizon. The NHCPP $\{N(t), t \geq 0\}$ has rate of arrivals $\lambda(t)$ on the time interval $t \in[0, T]$. A sample path of a NHCPP emulates two main properties in the proposed LEE: (1) random time of arrival, $0 \leq \tau_{1} \leq \tau_{2} \leq \ldots$ : these are the time instances corresponding to change in the degradation level of an airfoil aerodynamic properties (so-called shocks), and (2) magnitude of change $\left\{Y_{n}, n \geq 1\right\}$ in the degradation level of an airfoil aerodynamic properties at the $n^{\text {th }}$ time of arrival. In practice, for a probabilistic assessment, many NHCPP paths are sampled. The model incorporates one or more zones (adjacent or disjoint) along the span of the blade(s) affected by erosion, but are assumed to undergo independent degradation processes. The NHCPP modify the definition of a Poisson process so that it can incorporate time-dependent rate. The non-homogeneous Poisson process is suitable given the fact that the rate of LEE is not constant in time (timedependent arrival rates); on the short time scales (hourly), it is dependent on the rotor speed due to variations in the wind speed, while on the longer time scales it is dependent on seasonal variations such as rain intensity, and changes in environmental temperatures. We thus infer that the rate of occurrence of shocks is periodic, as shown in Figure 5. In addition, LEE consists of limited and finite number of stages resulting in varied and non-stationary incremental effects (severity) on the airfoils aerodynamic polars. It is in fact pragmatic to assume that the severity of LEE has a finite number of stages, because these could be categorised (e.g. via field observations), and their impact on aerodynamic properties emulated and quantified via measurements in wind tunnels as demonstrated in [6]. The impact of LEE on the lift and drag coefficients of the NACA64618 airfoil $[11,14,73]$ for 10 severity classes is shown in Figures 3 and 4.

The non-homogeneous Poisson process is similar to an ordinary Poisson process, except that the average rate of arrivals per period $\lambda(t)$ is allowed to vary with time (Figure 5). A classic definition of the Non-Homogeneous Poisson Process is formulated 


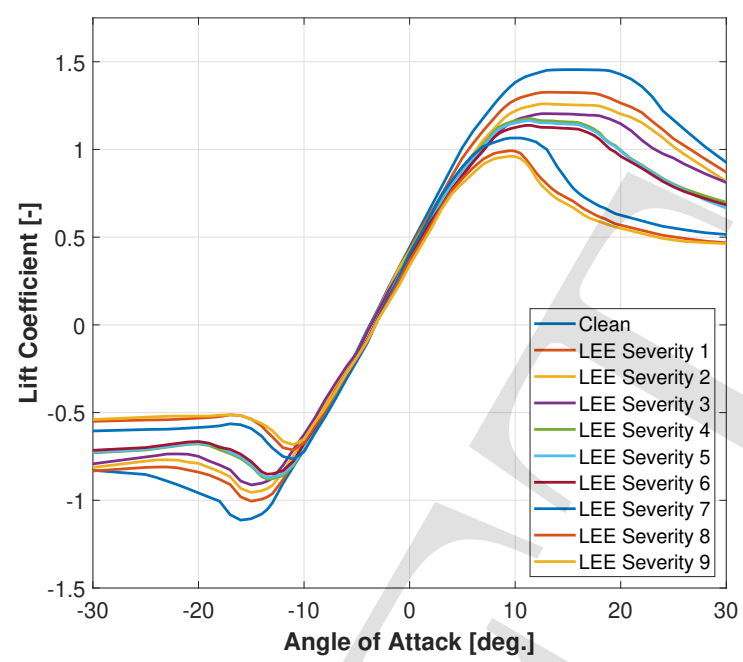

Figure 3. Lift coefficients for NACA64618 for 10 severity classes of leading edge erosion.

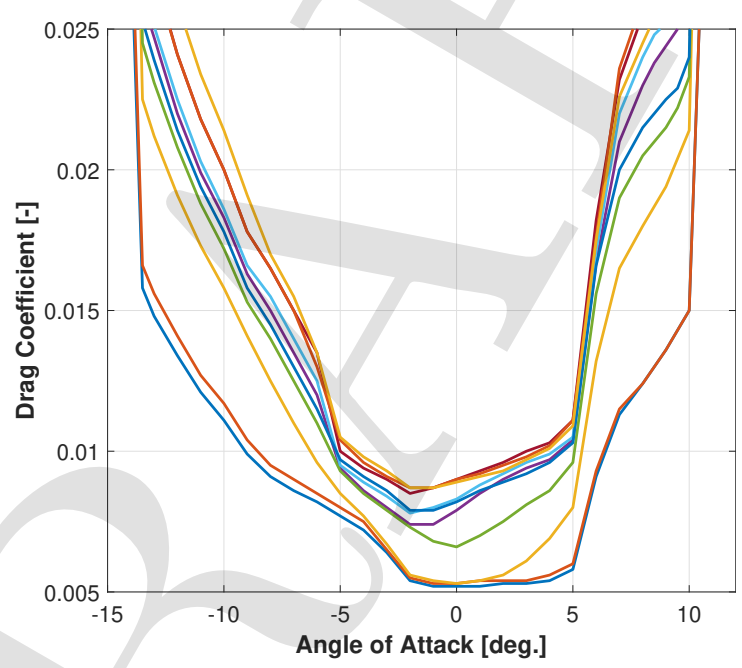

Figure 4. Drag coefficients for NACA64618 for 10 severity classes of leading edge erosion.

as follows [74]. Let $\lambda(t):[0, \infty) \rightarrow[0, \infty)$ be an integrable function; the counting process $\{N(t), t \geq 0\}$ is called a non-homogeneous Poisson process with rate $\lambda(t)$ if all the following conditions hold:

1. $\quad N(0)=0$

2. $\forall t, s \geq 0$, and $0 \leq u \leq t, N_{t+s}-N_{t}$ is independent of $N_{u}$

3. $\forall t, s \geq 0, P\left(N_{t+s}-N_{t}=0\right)=1-\lambda(t) s+o(s)$

4. $\forall t, s \geq 0, P\left(N_{t+s}-N_{t}=1\right)=\lambda(t) s+o(s)$, and

5. $\forall t, s \geq 0, P\left(N_{t+s}-N_{t} \geq 2\right)=o(s)$

where, $s$ is a a very short interval of time. Here $o(s)$ is a function that is negligible compared to $s$, as $s \rightarrow 0$. Assuming $o(s)=g(s)$, then:

$$
\lim _{x \rightarrow 0} \frac{g(s)}{s}=0
$$

One approach for generating Non-Homogeneous Poisson processes is the "process analogue" of acceptance-rejection called thinning, which is the scheme we adopt in our model. The procedure is as follows: 


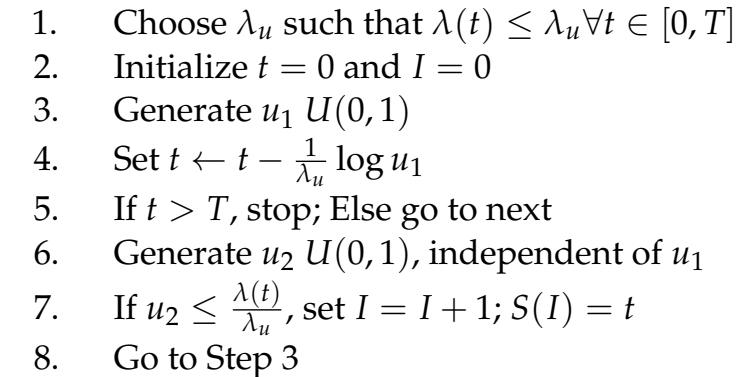

In the above procedure, the ratio $\frac{\lambda(t)}{\lambda_{u}}$ is the so-called thinning probability. In our model $\lambda_{u}$ is chosen as the maximum of the rate function $\lambda(t)$ (e.g. see Figure 5). For long degradation periods, it might be necessary to break $[0, T]$ into small intervals and pick a $\lambda_{u}$ for each interval in order to avoid a high rejection rate. This is known as piece-wise thinning. At the end of this procedure, the event times $S(I)$ (arrival times) and the counting process are yielded according to the Non-Homogeneous Process. Finally, for a non-homogeneous Poisson process with rate $\lambda(t)$, the number of arrivals in any interval is a Poisson random variable; however, its parameter can depend on the location of the interval. More specifically, we can write:

$$
N(t+s)-N(t) \sim \operatorname{Poisson}\left(\int_{t}^{t+s} \lambda(\alpha) d \alpha\right)
$$

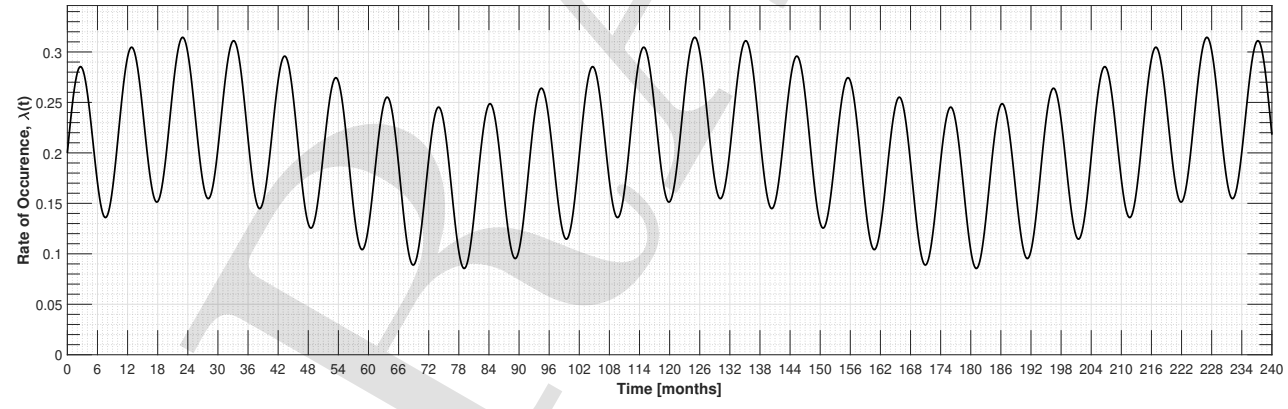

Figure 5. Cyclic rate function $\lambda(t)$ for the non-homogeneous Poisson process, representing the effect of weekly and seasonal variations on the rate. $\lambda(t)$ represents the rate of change in damage of LE erosion per given period.

Introducing the compounding effect to the Non-Homogeneous Poisson Process yields the so-called Non-Homogeneous Compound Poisson Process (NHCPP). The Compound Poisson Process, which is a very useful extension of a Poisson process, replaces the unit jumps of a Poisson process with random jump sizes [75]. The jumps magnitude at the $n^{\text {th }}$ arrival time has value $Y_{n}, n \geq 1$ attached to it. The successive $Y_{n}$ are assumed to be independent and identically distributed positive random variables each having the same distribution, and are also assumed independent of the underlying Poisson counting process of shocks. We assume that $Y=\left(Y_{1}, Y_{2}, \ldots\right)$ is a sequence of independent, identically distributed, real-valued random variables, and that $Y$ is independent of the underlying Poisson process. The compound Poisson process associated with the given Poisson process $\{N(t), t \geq 0\}$ and the sequence $\boldsymbol{Y}$ is the stochastic process $Z=\left\{Z_{t}, t \geq 0\right\}$, where:

$$
Z_{t}=\sum_{n=1}^{N(t)} Y_{n}
$$

The damage is always positive, i.e., that $P\left(Y_{n} \geq 0\right)=1$, and the damage accumulates additively according to $Z_{t}$ in Equation 2. In our model $Z_{t}$ represents the cumulative 
amount of erosion incurred on the blade section at time $N(t)$. In our model, we categorised 10 erosion severity levels reflected in the lift and drag coefficients, as shown in Figures 3-4 (other categorization could be specified as needed). The final level of degradation leads to the loss of function of the blade, not in the sense of a catastrophic loss (e.g. rupture), but rather implying that the blade loses its ability to generate lift efficiently. At this stage, we assume that once the blade section has reached level 9 of erosion, the process stops under the assumption that any further degradation becomes limited, since all the surface protective coatings and paint have already delaminated. We adopt a classical approach whereby $Y_{1}, Y_{2}, \ldots, Y_{n}$ are each exponentially distributed according to density:

$$
g_{Y}(y)=\mu \exp ^{-\mu y}
$$

where $\mu$ is the mean jump (shock) magnitude. It follows that the sum $Y_{1}+Y_{2}+\ldots+Y_{n}$ is Gamma distributed $G^{(n)}(z)$. For details, we refer the interested reader to [76,77]. Formally, we adopt a truncated exponential distribution for $Y_{n}$ in Equation 3. The logic being that large erosion magnitude jumps (e.g. large jumps in erosion severity) in one shock event are physically hard to justify. As a result, the upper truncation limit for the shock is set to a magnitude of 4 in our model. Furthermore, as more events arrive between $[0, T]$, and damage is compounded $Y_{1}+Y_{2},+\ldots$, we adjust the upper truncation limit for the shock magnitude from 4 to 1 when $\left\{I \geq 3 \wedge\left(Z_{\max }-\sum_{n=1}^{N(t)=I} Y_{n}\right) \leq 3\right\}$. Finally, sampled jumps of magnitude $Y_{n}>0$ for $\tau_{n}<T$ are not allowed once the compounded damage has reached its highest compounded severity class $\left(Z_{\max }=9\right)$ before the end of time horizon $T$ has been reached. This is reasonable as any additional shocks (e.g. rain or hail) cannot not incur any additional erosion, as there are not any more surface materials that can be easily eroded.

Finally, we introduce wind speed and blade location dependencies to the model. For a given time of arrival of a shock, our model associates higher damage (1) in the event when the shock is concurrent with higher inflow wind speeds due to higher momentum (and thus kinetic energy) of the impacting particles anywhere on the blade and (2) in the outboard sections of the blade due to the local higher relative speed of the flow. We require the mean jump (shock) magnitude $\mu$ to comprise a linearly increasing function of blade radial location and quadratic with wind speed. The final outcome is thus an NHCPP degradation paths as shown in Figure 6, where 5 zones along the span of blade 2 are chosen to undergo leading edge degradation. Even though the exact pattern and location of surface changes during operation is a random process, we see that our model tends to provide higher damage rates of the leading edge on the outboard sections of the blade compared to the inboard sections.

\subsection{Stochastic aerodynamic Polars model}

In this section, we generate a stochastic ensemble of degrading airfoil aerodynamic polars for use in forward aero-servo-elastic simulations. The outputs of the NHCPP are the degradation paths describing different degradation zones along the span of the rotor blades. Each arriving shock at a given time along these paths corresponds to a jump of a certain magnitude in the leading edge erosion severity. A jump in the erosion severity corresponds implicitly to a degradation of the aerodynamic properties of the airfoils affected by erosion along the span of the blade(s). However, uncertainties in the degrading airfoil aerodynamics lift and drag coefficients curves still remain to be accounted for. The stochastic aerodynamic Polars model, that we here describe, accounts for such uncertainties by parameterizing the lift and drag coefficients curves. Considerable effort and capital is invested in predicting the static aerodynamic lift $C_{L}$, drag $C_{D}$ and moment $C_{M}$ coefficients of airfoils as accurately as possible under various operating conditions. The lift and drag coefficients are then used by the wind turbine designers 


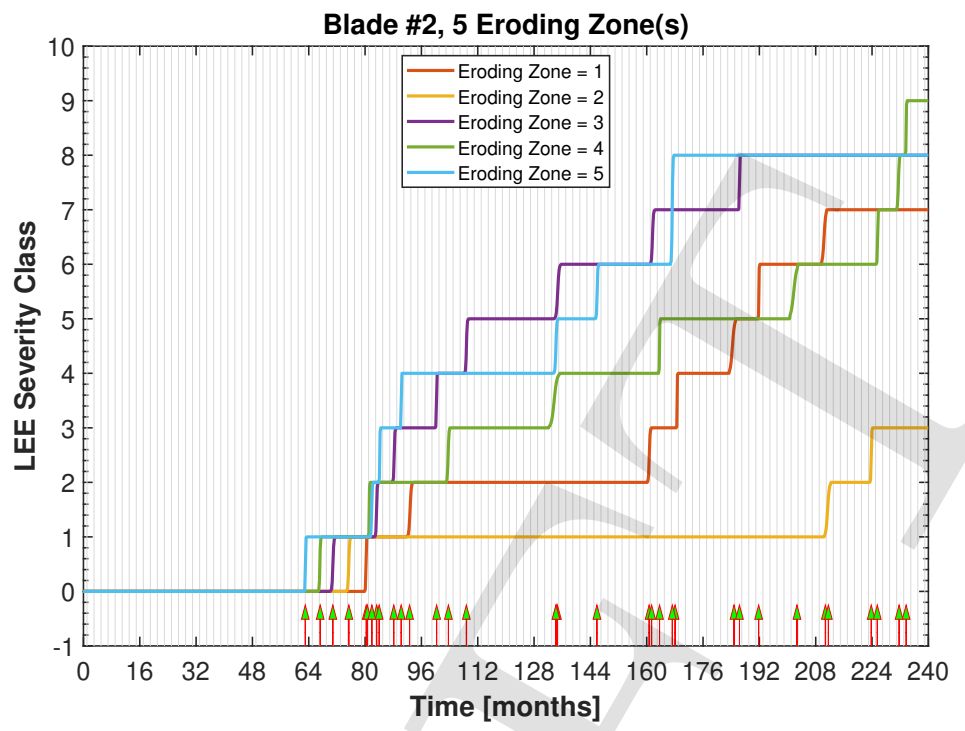

Figure 6. A sample of the NHCPP of the leading edge erosion on 5 zones along the span of blade 2 over a 20 -year period ( 240 months). Section 5 is the outermost section along the span of the blade.

as input to aero-servo-elastic simulations to predict power performance, extreme and fatigue loads in addition to stability margins in normal and extreme operating and stand still conditions. An airfoil's static aerodynamic data are almost exclusively derived from measurements acquired in wind tunnel tests. However, an airfoil section on the wind turbine rotor operates in 3-dimensional, un-steady and turbulent inflow under the guidance of a control system, none of which are accounted for in static wind tunnel tests. Some aspects of uncertainty in airfoil data (surface roughness, 3D corrections, effect of Re numbers, wind tunnel measurements or geometric distortions) have been studied in [72]. The general consensus is that uncertainties in airfoil data do affect a wind turbine's performance and structural loading. Consequently, our proposed LEE model also takes into account the inherent uncertainty in airfoil static lift and drag coefficients during the erosion period via a stochastic model of static airfoil lift and drag polar curves. The details of this model can be found in Abdallah et al. [72], with a short summary presented herein for conciseness.

A well known result from Blade Element Moment theory links the aerodynamic lift $\left(C_{L}\right)$ and drag $\left(C_{D}\right)$ coefficient to axial $(a)$ and tangential $\left(a^{\prime}\right)$ induction factors, as follows:

$$
\begin{aligned}
\frac{a}{1-a} & =\frac{\sigma^{\prime}\left(C_{L} \cos \phi+C_{D} \sin \phi\right)}{4 \sin ^{2} \phi} \\
\frac{a^{\prime}}{1-a} & =\frac{\sigma^{\prime}\left(C_{L} \sin \phi-C_{D} \cos \phi\right)}{4 \lambda_{r} \sin ^{2} \phi}
\end{aligned}
$$

where $\sigma^{\prime}$ is the rotor solidity, $\Phi$ is the angle of the incoming relative wind with the rotor plane, $\lambda=\frac{\omega r}{V_{0}}, \omega$ is the rotor speed, $r$ is the radial distance from the rotor center, and $V_{0}$ is the freestream wind. Our approach to affecting aerodynamic induction is by introducing a stochastic model of the lift and drag coefficients curves in BEM.

Several sources of uncertainties affect the lift and drag coefficients. These uncertainties associated with assessment of airfoil characteristics in wind tunnels, uncertainties due to 3D flow correction, uncertainties stemming from surface roughness, uncertainties related to the blade geometric distortions in manufacturing and handling, uncertainties related to the blade geometric distortions when deflected under load, uncertainties due to the effects of Reynolds number, uncertainties associated with extending airfoil 
aerodynamic characteristics to post stall, and finally uncertainties stemming from the validation of airfoil data by field full scale prototype test. It is not possible to quantify the joint distribution of all these random variables and as a result a more simplified approach is chosen via a stochastic model as proposed in [72]. The stochastic model consists in parameterizing the lift coefficient curve by the slope in the linear range $\frac{\partial C_{L}}{\partial \alpha}$, the point indicating the start of the trailing edge separation $\left(A \circ A_{T E S}, C_{L, T E S}\right)$, the point of maximum lift $\left(A o A_{\max }, C_{L, \max }\right)$ and the point where the stall recovery is initiated $\left(A o A_{S R}, C_{L, S R}\right)$. The drag coefficient is parameterized by a bias at low angles of attack, and by the point where max drag coefficient occurs at $A o A= \pm 90^{\circ}$ where it exhibits the largest variations. Figure 7 shows samples of the stochastic lift and drag coefficients for $N A C A 64618$ for an erosion of severity class 5 . These perturbations result in modified $C_{L}$ and $C_{D}$ curves that maintain the primary characteristics of the original curve, but differ in both magnitude and feature location, emulating possible uncertainties affecting the aerodynamic properties of the airfoil section under real operation.

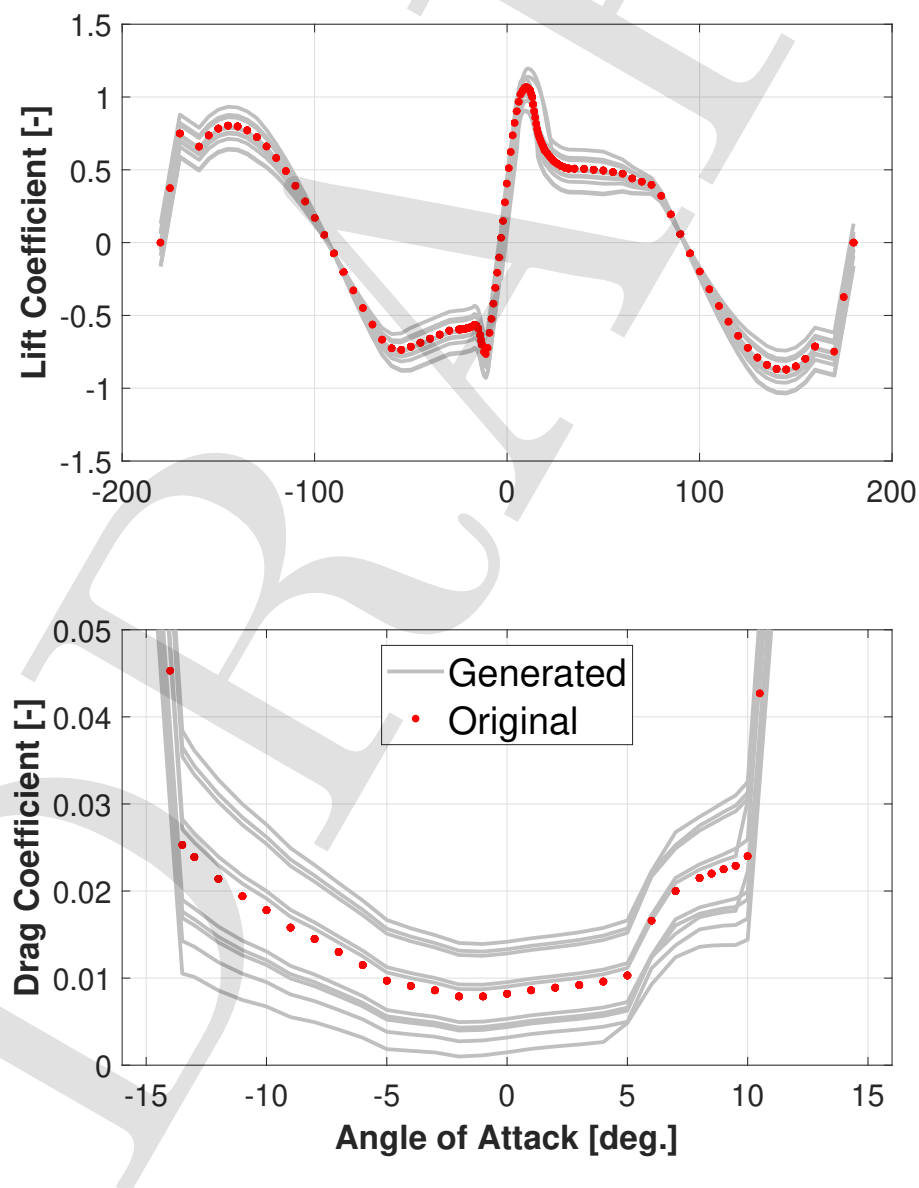

Figure 7. Samples of the generated stochastic lift and drag coefficients of airfoil NACA64618 corresponding to erosion severity class 5 .

Putting it all together, the NHCPP coupled to the generator of stochastic ensemble of aerodynamic polars yields realistic degrading aerodyanmic polars over the degradation time horizon, for every degrading zone and airfoil section on the rotor blades. For example, Figure 8 shows the degrading $C_{L}$ over a 20-year period (240 months) for for NACA64618 airfoil. Figures 9-11 represent the corresponding degrading max lift coefficient, degrading angle of attack at max lift coefficient and degrading lift coefficient at $\alpha=5^{\circ}$, respectively. 


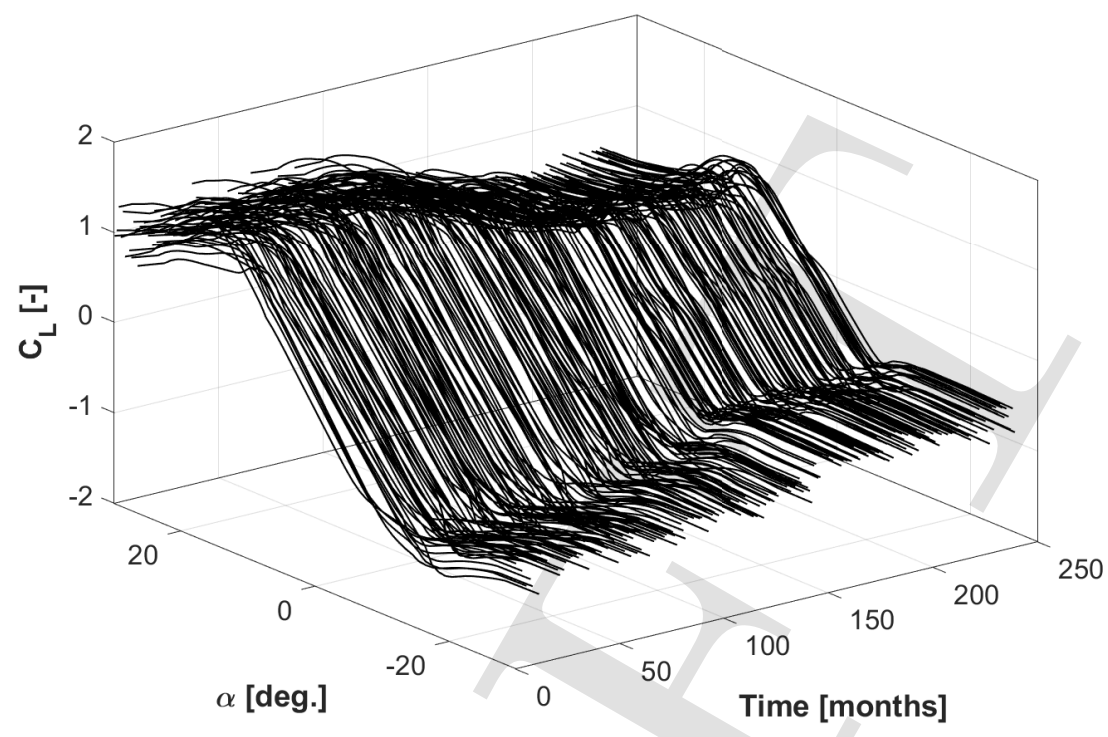

Figure 8. NHCPP generated degrading static $C_{L}$ for NACA64618. This is used as input to the aeroelastic simulations for Blade 1 , section 5 . For each pre-selected degrading section on a blade a set of such curves are generated.

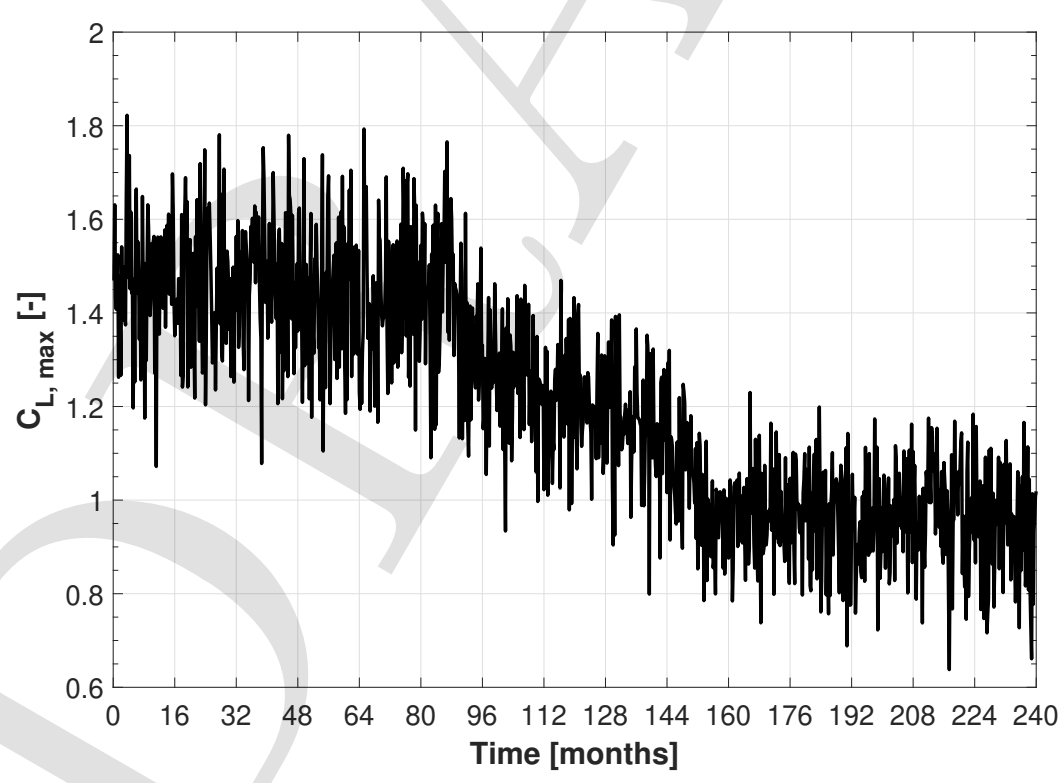

Figure 9. Degradation of the max lift coefficient for Blade 1, section 5.

\subsection{Overview of the Algorithm}

The main ingredients of the model include a (1) spatio-temporal stochastic damage process and (2) a generator of stochastic ensemble of degrading airfoil aerodynamic polars. The algorithm is summarized in Algorithm 1.

\section{Uncertainty Modelling and Aeroelastic Simulations}

Two categories of uncertain random variables (RV) are considered: wind inflow and aerodynamic effects. We have elaborated on the aerodynamic uncertainties in the 


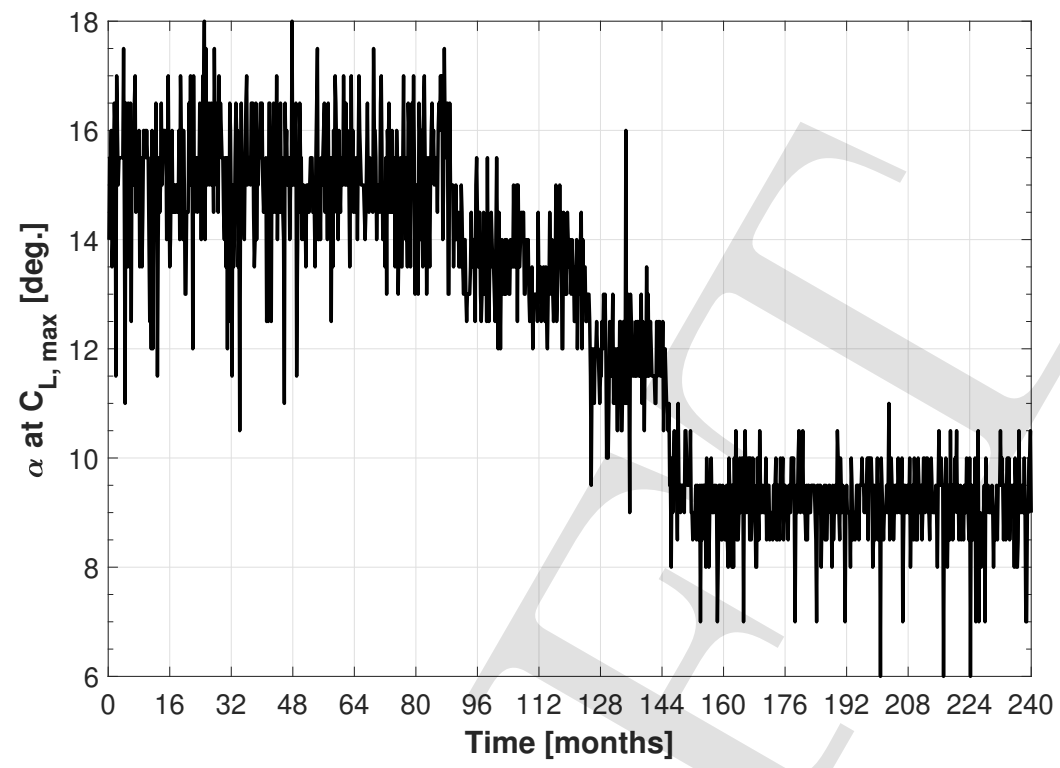

Figure 10. Degradation of angle of attack at max lift coefficient for Blade 1, section 5.

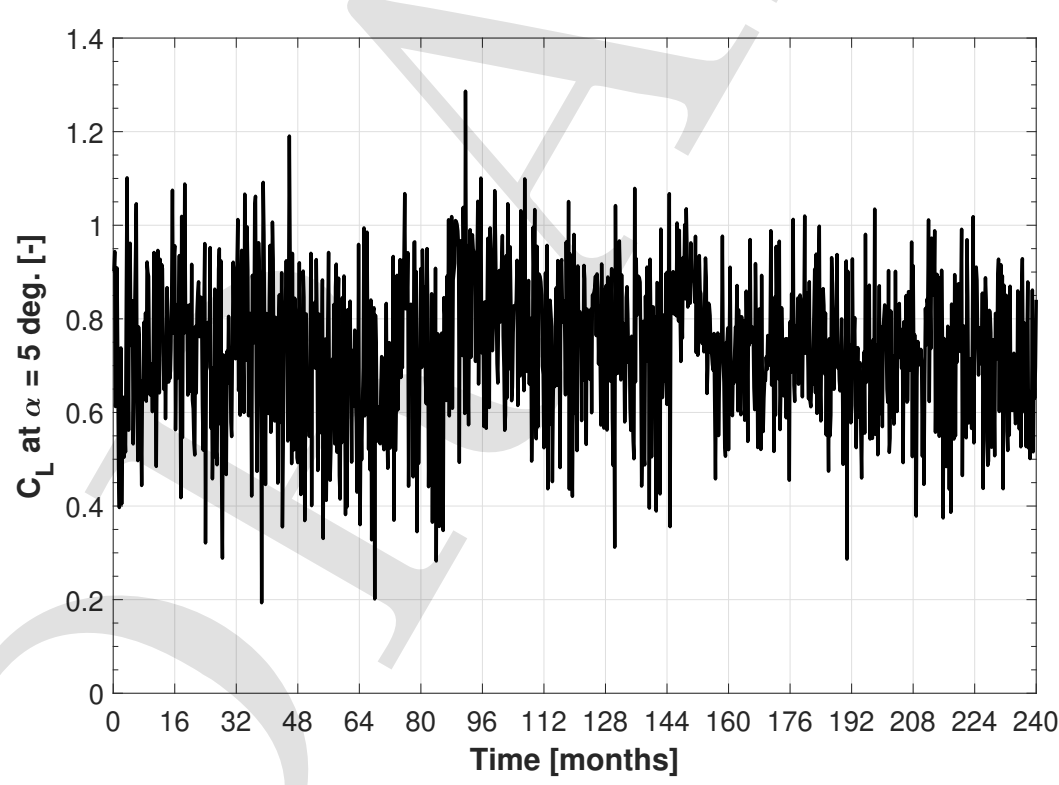

Figure 11. Degradation of the lift coefficient at $\alpha=5^{\circ}$ for Blade 1 , section 5 .

previous section. In this section, we detail the wind inflow uncertainties and the aeroelastic simulations setup. The conventional wind turbine structural damage computation process utilizes aeroelastic load simulations for wind turbines under normal and extreme operation and wind inflow turbulence conditions, whereby the extreme and fatigue load cycles determined over a short period of time at each mean wind speed are extrapolated over the full expected lifetime. Such a process does not consider the changes in inflow and environmental changes over long time periods, indeed relevant to LE erosion process. 


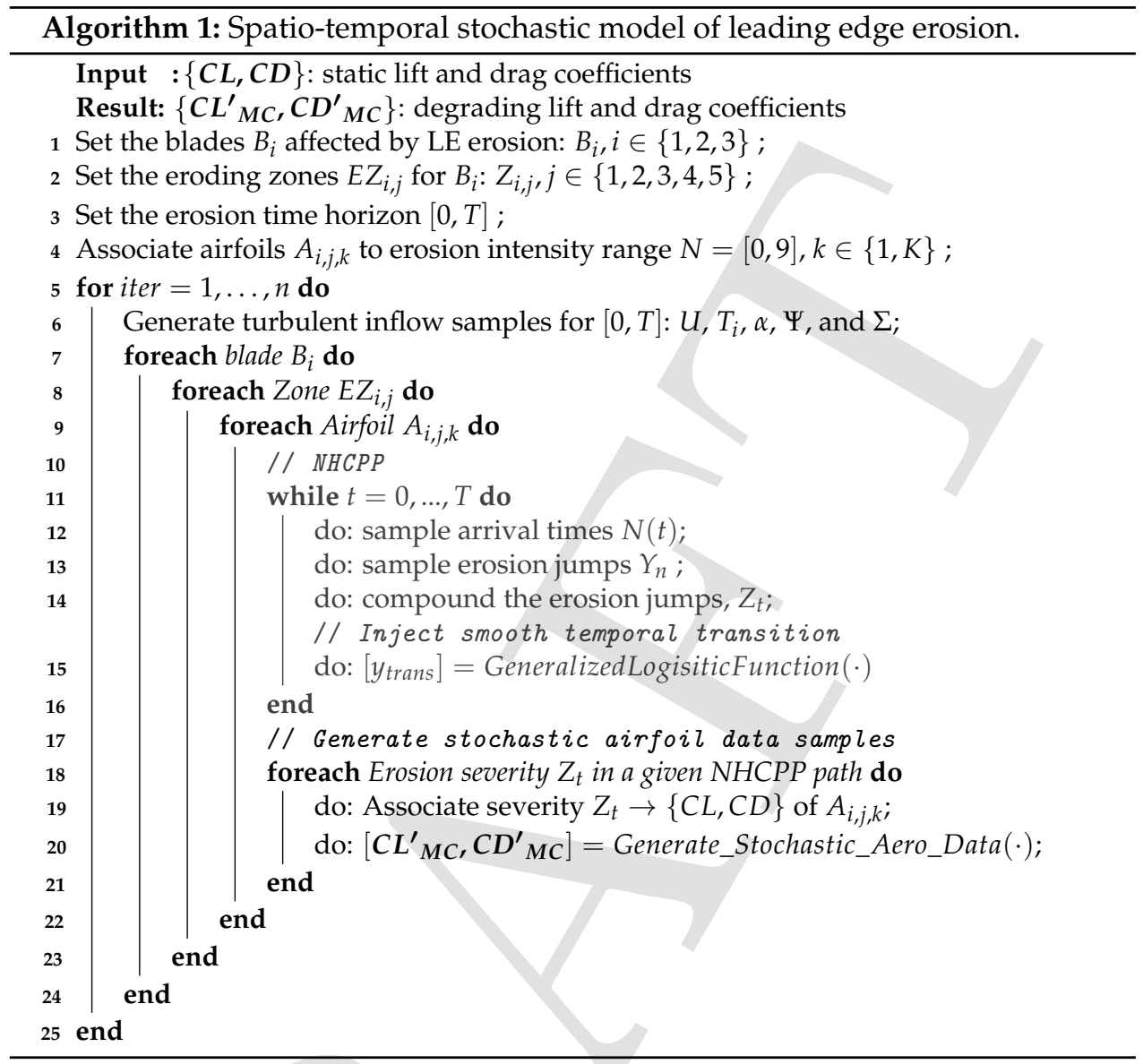

\subsection{Inflow RVs and their Stochastic Models}

The variation in the structural dynamic response of wind turbines is significantly dependent on the turbulent inflow wind field conditions, including the mean wind speed, turbulence, wind shear, and inflow skewness. In accounting for these influences, we introduce the following RVs in the simulations setup: Mean wind speed, $U$, turbulence intensity, $T_{i}$, wind shear, $\alpha$, horizontal inflow skewness, $\Psi$, and the vertical inflow skewness, $\Sigma$.

The mean wind speed follows a Weibull distribution $U \sim \mathcal{W B} \mathcal{L}\left(A_{U}, K_{U}\right)$, truncated to $[4-25] \mathrm{m} / \mathrm{s}$, with parameters specified as follows:

$$
\begin{aligned}
\mathbb{E}(U) & =8.5, \text { where } A_{U}=\frac{2 \times \mathbb{E}(U)}{\sqrt{\pi}} \\
K_{U} & =2.0
\end{aligned}
$$

The conditional dependence between the turbulence $\sigma_{U}$ and the mean wind speed $U$ is defined in the Normal Turbulence Model described in the wind turbine design standard [78]. Here, we elect to use a reference ambient turbulence intensity $I_{\text {ref }}=.16$ (the expected value of the turbulence intensity at $15 \mathrm{~m} / \mathrm{s}$ is called $\left.I_{\text {ref }}\right)$. This dependency is given by the local statistical moments of $\sigma_{u} \sim \mathcal{L} \mathcal{N}\left(\mu_{\sigma_{U}}, \sigma_{\sigma_{u}}^{2}\right)$ as:

$$
\begin{aligned}
\mathbb{E}\left(\sigma_{u} \mid U\right) & =I_{\text {ref }}(0.75 u+3.8) \\
\mathbb{V}\left(\sigma_{u} \mid U\right) & =\left(1.4 I_{\text {ref }}\right)^{2}
\end{aligned}
$$


As a result, the turbulence intensity is expressed as: $T_{i}=\frac{\sigma_{u}}{u}$.

The wind profile above ground level is expressed using the power law relationship, which defines the mean wind speed $u$ at height $Z$ above ground as a function of the mean wind speed $u_{h}$, measured at hub height $Z_{h}$ as reference:

$$
\frac{u}{u_{h}}=\left(\frac{Z}{Z_{h}}\right)^{\alpha}
$$

where $\alpha$ is a constant called the shear exponent. The conditional dependence between the wind shear exponent $\alpha \sim \mathcal{N}\left(\mu_{\alpha}, \sigma_{\alpha}^{2}\right)$ and the mean wind speed $U$ is given by [79]:

$$
\begin{aligned}
& \mathbb{E}(\alpha \mid U)=0.088(\ln (u)-1) \\
& \mathbb{V}(\alpha \mid U)=\left(\frac{1}{u}\right)^{2}
\end{aligned}
$$

We define a custom conditional dependence between the inflow horizontal skewness $\Psi$ and the mean wind speed $U$, truncated to $[-11,11]$ deg., $\Psi \sim \mathcal{N}\left(\mu_{\Psi}, \sigma_{\Psi}^{2}\right)$ :

$$
\begin{aligned}
& \mathbb{E}(\Psi \mid U)=\ln (u)-3 \\
& \mathbb{V}(\Psi \mid U)=\left(\frac{15}{u}\right)^{2}
\end{aligned}
$$

The conditional dependence between the inflow vertical skewness $\Sigma$ and the mean wind speed $U$ and turbulence intensity $T_{i}$, truncated to $[-6,6] \mathrm{deg}$., is designed such as:

$$
\Sigma \sim \mathcal{N}\left(\mu_{\Sigma}, \sigma_{\Sigma}^{2}\right): \begin{cases}\mu_{\Sigma}=-2, \sigma_{\Sigma}^{2}=1.0, & \text { if } T_{i} \leq 10 \% \& \alpha \leq 0.1 . \\ \mu_{\Sigma}=1.5, \sigma_{\Sigma}^{2}=1.0, & \text { otherwise. }\end{cases}
$$

The blade leading edge degradation scenario occurs over a 240-months period; consequently, we introduce an additional element of uncertainty by allowing a timevarying aspect of the inflow conditions, namely, the mean wind speed $\mathbb{E}(U)$, the shape parameter $K_{U}$ of the Weibull distribution, and the reference ambient turbulence intensity $I_{\text {ref }}$ as shown in the example in Figure 12.

We choose to sample the wind inflow and the aerodynamic RVs using the Sobol Quasi-Random sequences, which are designed to generate samples that are uniformly distributed over the unit hypercube, i.e., as uniformly as possible over the multidimensional input space [80]. In total, we sample 1200 joint samples of the wind inflow RVs over the degradation period, as shown in Figures 13 - 14. A sample of $U$, $\sigma_{U}, \alpha, \Psi$ and $\Sigma$ is used to generate a realization of inflow turbulent wind field time series, combined with a sample of stochastic and degrading $C_{L}$ and $C_{D}$ form the input to the OpenFAST aero-servo-elastic environment to simulate the corresponding aeroelastic response of the wind turbines structure under continuous erosion of the leading edge of the blades.

\subsection{Aero-servo-elastic Simulations Setup}

Our coupled aero-servo-elastic simulations with the proposed NHCPP leading edge degradation model setup is based on the OpenFAST simulator. OpenFAST [81], is a coupled aero-hydro-servo-elastic analysis tool for modeling wind turbines. The 


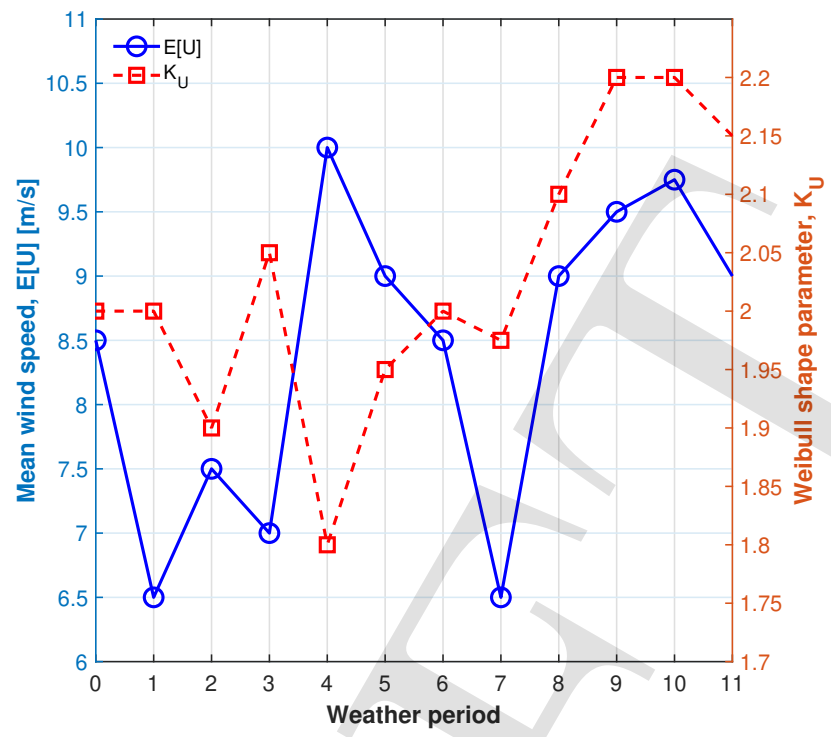

(a)

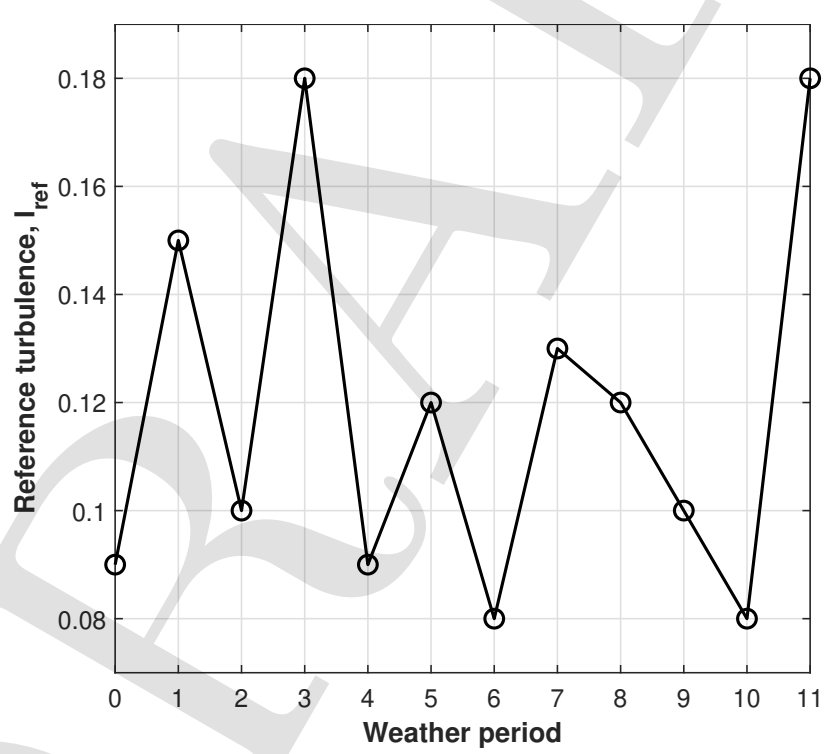

(b)

Figure 12. Long term inflow variations: (a) Varying mean wind speed and shape parameter $K_{U}$ of the Weibull distribution. (b) Varying reference turbulence Iref.

primary use of OpenFAST is to run nonlinear time-domain simulations. The simulations in OpenFAST considered the reference NREL three-bladed up-wind, horizontal-axis wind turbines with $126 \mathrm{~m}$ rotor diameter, $5 \mathrm{MW}$ rated power and hub height of $90 \mathrm{~m}$. The layout of the airfoils along the span of the blade are shown in Figure 15. We also list some of the more important properties of the simulated wind turbine in Table 3.

OpenFAST is a wind-turbine-specific nonlinear time-domain simulator that employs a combined modal and multibody dynamics formulation, adopting limited degrees of freedom (DOF). Large structural elements (blades and tower) are characterized by properties such as stiffness and mass per unit length, which account for flexibility characteristics. OpenFAST models the turbine dynamics using 24 degrees of freedom (DOFs), including two blade-flap modes and one blade-edge mode per blade, two fore-aft and two side-to-side tower bending modes, nacelle yaw, the generator azimuth angle and the compliance in the drive train between the generator and hub/rotor. The aerodynamic model is based on the unsteady Blade Element Momentum theory, including skew 


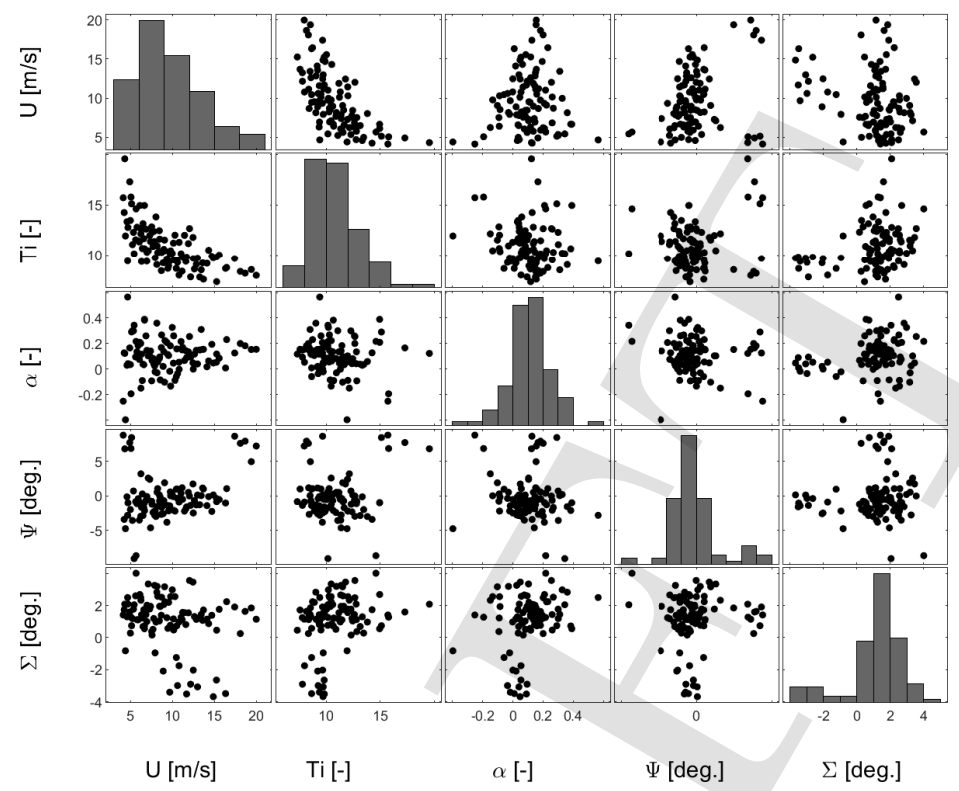

Figure 13. Joint samples of the environmental sources of uncertainties for degradation period 0. $\mathbb{E}(U)=8.5 \mathrm{~m} / \mathrm{s}, K_{U}=2.0, I_{\text {ref }}=0.09$.

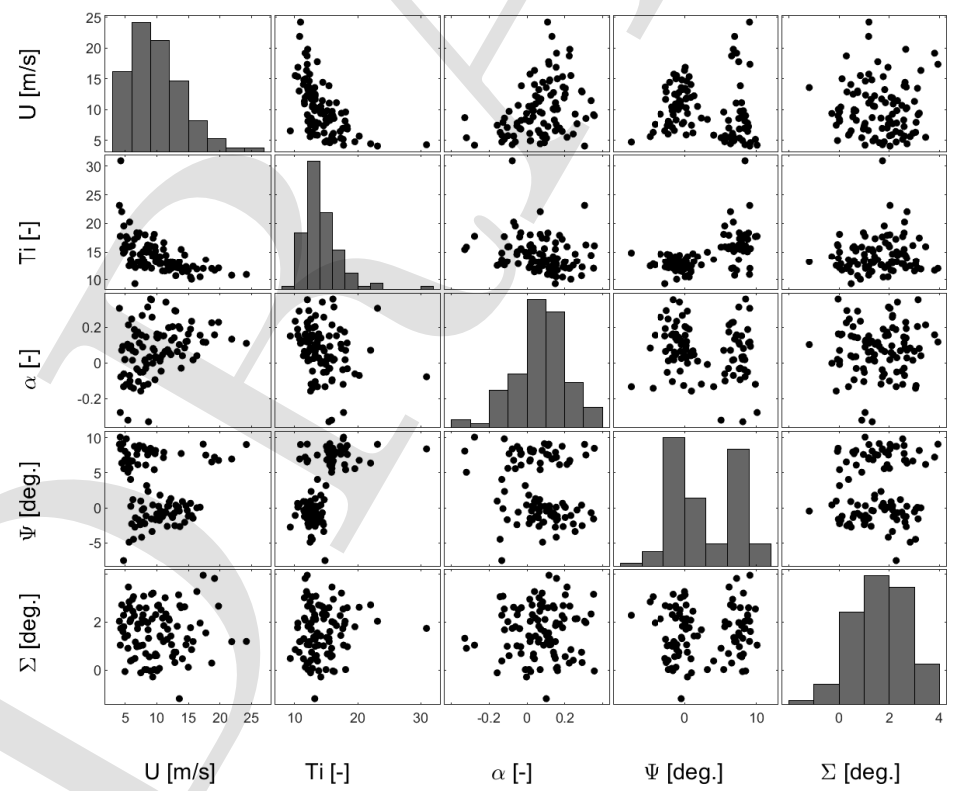

Figure 14. Joint samples of the environmental sources of uncertainties for degradation period 5.

$$
\mathbb{E}(U)=9.0 \mathrm{~m} / \mathrm{s}, K_{U}=1.95, I_{\text {ref }}=0.12 \text {. }
$$

inflow, dynamic stall and generalized dynamic wake. The Blade aerodynamic polars are provided as a-priori input and are used as lookup tables or for interpolation. In our case, the aerodynamic polars correspond to the degrading lift and drag coefficients generated in the NHCPP, such as the one shown in Figure 8. The stochastic input wind field uses the Kaimal turbulence model [82]. A common practice is to generate a significant number of stochastic simulations for various operating and environmental conditions in order to cover variability on aeroelastic fatigue and extreme load analysis due to the stochastic nature of the input wind field and variability in weather patterns over long time horizons ( $>$ 1-year). 


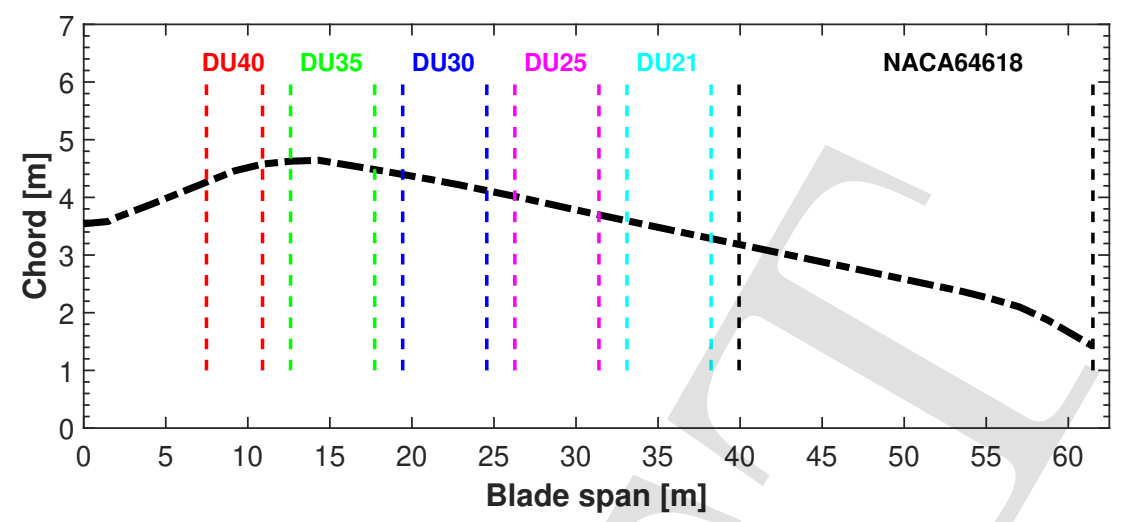

Figure 15. Distribution of airfoils along the span of the blade. Example of the NREL5MW reference wind turbine.

Table 3: Properties of the NREL 5-MW reference wind turbine.

\begin{tabular}{ll}
\hline Number of blades & 3 \\
Rotor diameter & $126 \mathrm{~m}$ \\
Hub height & $90 \mathrm{~m}$ \\
Rated power & $5 \mathrm{MW}$ \\
Cut-in wind speed & $3 \mathrm{~m} / \mathrm{s}$ \\
Cut-out wind speed & $25 \mathrm{~m} / \mathrm{s}$ \\
Control & Variable Speed, Collective Pitch \\
Variable speed & from cut-in to cut-out wind speed \\
Variable pitch & from cut-in to cut-out wind speed \\
Rated wind speed & $11.4 \mathrm{~m} / \mathrm{s}$ \\
Cut-in and rated RPM & $6.9-12.1 \mathrm{RPM}$ \\
\hline
\end{tabular}

In our simulation setup, we assign a maximum of 5 independent zones that could undergo leading edge erosion per blade. The location (normalized by the blade length) of the degradation zones along the span of the three blades are:

$$
E Z:[0.65,0.68],[0.70,0.80],[0.82,0.85],[0.87,0.90],[0.92,1.00]
$$

We simulate 20 years (240 months) worth of wind turbine operation with blades LEE. We generate one sample every (approximately) six days, resulting in a total of 1200 simulated samples. Every sample is a 600-second multi-variate time series with 228 sensor signals each, sampled at $100 \mathrm{~Hz}$. Few examples of the time series signals are shown in Figures 16- 18.

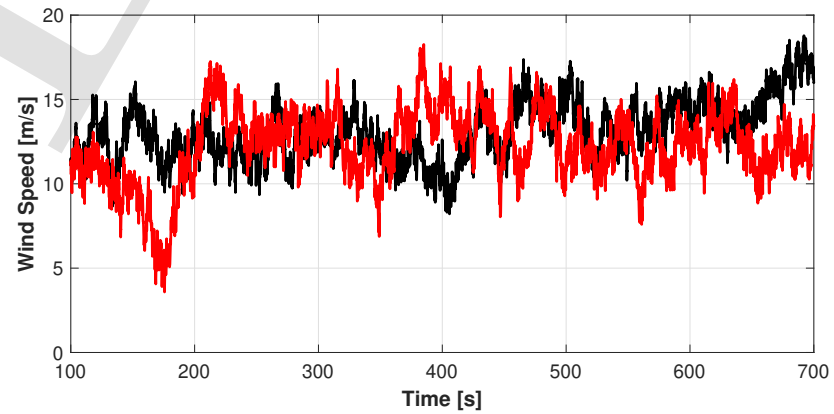

Figure 16. Sample hub height wind speed. (Black) beginning and (Red) end of degradation period. 


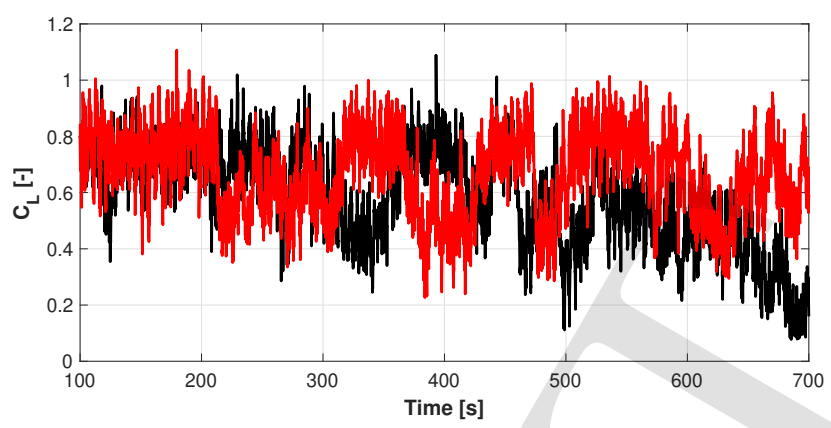

Figure 17. Sample lift coefficient at the airfoil profile corresponding to $95 \%$ of the span of blade 1 . (Black) beginning and (Red) end of degradation period.

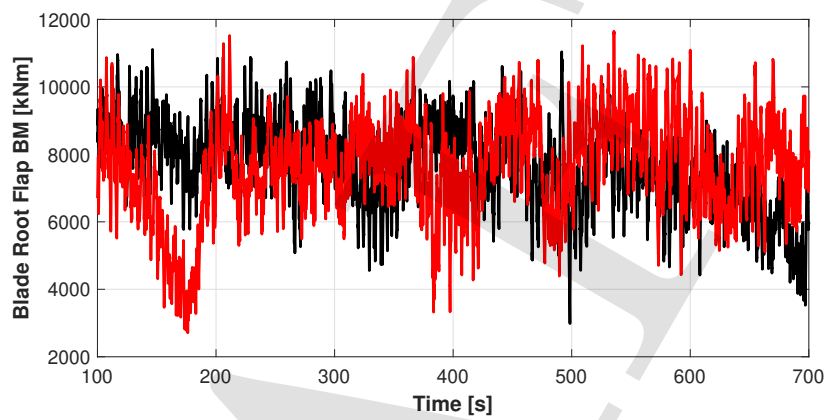

Figure 18. Sample flapwise bending moment at the root of blade 1. (Black) beginning and (Red) end of degradation period.

\subsection{Retained Output from Aero-servo-elastic Simulations}

As mentioned in the beginning of the paper, the motivation for this research is the Aerosense research project, where we are developing a novel MEMS-based aerodynamic surface pressure and aero-acoustic smart measurement system for wind turbines blades, for use in Structural Health Monitoring tasks. In this simulated setup, we assume one Aerosense node located at 0.96 of the blade length, located within eroding zone 5 (near the tip of the blade). From our aero-servo-elastic simulations, we elect to retain those output signals which are expected to emanate from the Aerosense measurements nodes, namely, lift and drag coefficients, angle of attack, and wind speed.

Table 4: Retained sensors output of aero-servo-elastic simulations.

\begin{tabular}{ll}
\hline Sensor name & Description \\
\hline Time & Time steps of the simulations \\
Wind 1 VelX & X-direction wind velocity at hub-height \\
B1N9Cl & Lift force coefficient at Blade 1, Aerosense Node at $0.96 R$ \\
B1N9Cd & Drag force coefficient at Blade 1, Aerosense Node at $0.96 R$ \\
B1N9Alpha & Angle of attack at Blade 1, Aerosense Node at 0.96R \\
\hline
\end{tabular}

\section{Diagnosing LEE via Transformers}

The simulation pipeline described above allows us to generate data which replicate the sensory output of Aerosense nodes. Thus, we are able to produce datasets to train a deep learning method, with the end goal being to detect and estimate the extent of leading edge erosion on wind turbine blades. We choose to implement a modified version of a popular class of sequential deep learning models: Transformers [41]. 
To motivate the diagnostics task, we consider the case where $C_{L}$ time series signals emanate from two sections along the span of the blade. Figure 19 shows a scatter plot of time series of $C_{L}$ at sections $0.96 R$ vs $0.75 R$ of the blade for wind speeds varying between $6-16 \mathrm{~m} / \mathrm{s}$ and no LEE (clean blade). Figure 20 shows a similar plot for a fixed wind speed $11 \mathrm{~m} / \mathrm{s}$ and evolving severity of leading edge erosion at section $0.96 R$. An important property is that the relational dependency of the $C_{L}$ between the two sections will change over time either due to changes in the severity of the leading edge erosion or due to short-term variation in inflow and operating conditions. The problem is thus to detect leading edge erosion and its severity, which could be regarded as a problem of finding the optimal discriminants for erosion versus other effects (inflow, operational conditions, aerodynamic uncertainties, etc.) from multivariate time-series response signals.

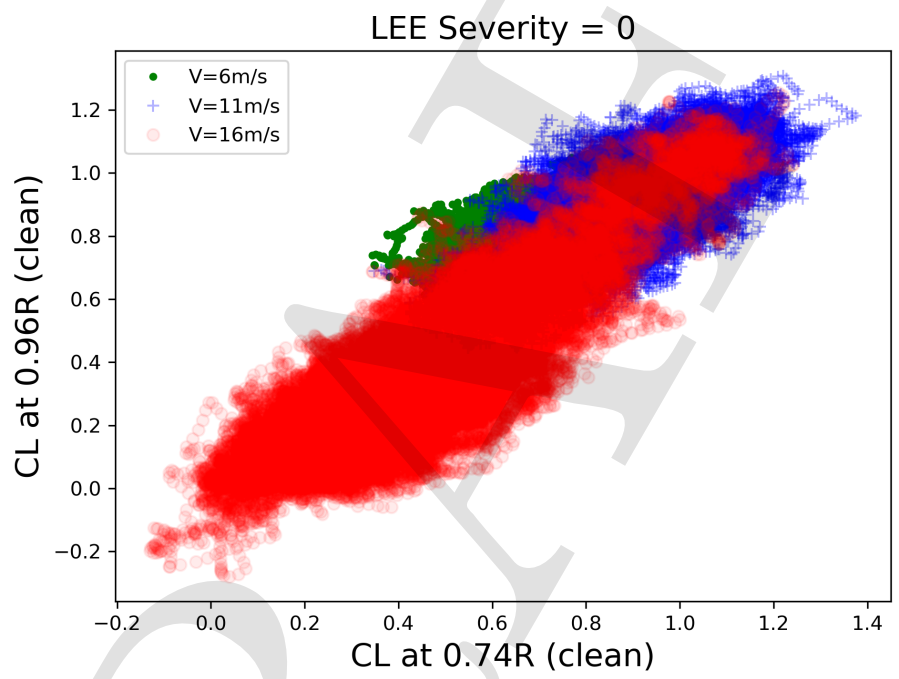

Figure 19. $C_{L}$ for wind speeds varying between $6-16 \mathrm{~m} / \mathrm{s}$ and no blade erosion.

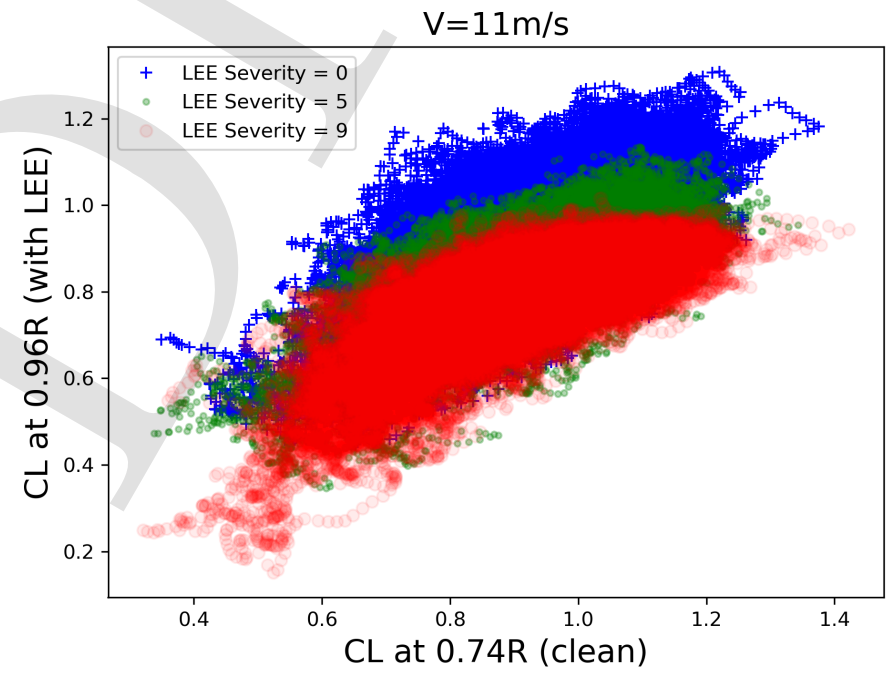

Figure 20. $C_{L}$ for fixed wind speed $U=11 \mathrm{~m} / \mathrm{s}$ and evolving severity of leading edge erosion.

The following are aspects we wish to build into the diagnostics method for LEE classification, taking into account the nature/circumstance of an operating wind turbine in the field: 
- Labeled data for LEE are hard to acquire and scarce; as a result, any method must be designed not to suffer from overfitting under scarce labeled data availability.

- Diagnostics shall be done with remote streaming of sensor data. Human intervention and turbine down-time should be alleviated.

- The data used in the diagnostics method shall replicate the sensory output of a single micro-electro-mechanical-systems based aerodynamic and aero-acoustic measurement node located near the tip of one wind turbine blade.

- The method shall be capable of ingesting 10 minute long multivariate time-series (industry standard SCADA recording length), sampled at $100 \mathrm{~Hz}$, resulting in $60^{\prime} 000$ data points long sequences. A sampling rate of $100 \mathrm{~Hz}$ may seem excessive, but the aim is to capture even small turbulence scales.

- Exploits a supervised pre-training scheme to initialize a Transformer-based network by utilizing large-scale labeled data from aeroelastic simulations of the turbine and a degradation model as presented earlier in the paper.

- Once pre-training is completed, the pre-trained model can be further adapted to the specific classification task by fine-tuning the model parameters on small-scale labeled data. In this way, the general knowledge and representations about LEE can be transferred to a label-scarce task, thereby improving the generalization performance of the model as well as reducing the risk of overfitting.

- Physics-constraints shall be built-in the loss or likelihood function.

- The output predictions should be probabilistic in nature.

\subsection{Experiments and Datasets}

We utilize multivariate time-series data generated via the coupled simulation pipeline previously described. The term multivariate is used in the sense that we are monitoring multiple signals pertaining to lift, drag, angle of attack and inflow velocity emanating from an Aerosense node located at one section at the tip of the blade. Multivariate could also be thought of in the sense that we can take advantage of the presence of multiple Aerosense stations at different locations along the span of the blade(s), however, this is beyond the scope of the current work. After some initial testing, we have opted to utilize the four variables shown in Table 4 as inputs to the Transformer. For instance, no performance benefit was found when including simulated acceleration data.

Three sets of experiments, each with different data requirements, are performed aiming to answer the following questions:

1. Are we able to learn the LEE severity classes from MTS data, in the general machine learning sense, with balanced data classes and no prior knowledge?

2. In a continuous monitoring context, are we able to diagnose jumps in LEE severity and therefore identify the degradation path that the system takes?

3. Are we able to do so in a realistic setting, with all previously described sources of uncertainty present in the simulations?

Thus, the goal of the first set of experimental tasks is to train the neural network on a generalized dataset with reduced uncertainty and where each degradation class appears in equal measure. This is not representative of a real degradation path, where the inherent stochasticity of the NHCPP may result in some classes appearing very briefly, but we aim to avoid biasing the network towards a random predominant class. Moreover, this set of experiments also aims to understand how data availability affects classification performance, and how separable the classes are. Two datasets, corresponding to experiments 1.1 and 1.2, are generated: one with the full 10 severity classes and one 
with a reduced amount of severities (levels 0, 1,6 and 9). For this set of experiments, no information on previous states is used. In each of the sub-experiments, 4800 aero-elastic simulations are gathered, then split into train, validation and test sets in a 70/20/10 split.

The second set of experiments aims to assess whether using full degradation paths in a continuous monitoring setup to train the diagnostics method is a suitable strategy. Here, the datasets are comprised of full NHCPP degradation paths, albeit with reduced uncertainty. Due to the stochastic degradation, the datasets do not have balanced classes. Assuming that a continuous monitoring system is in place, we therefore have access to the previous degradation states. This allows for degradation monotonicity to be enforced in the prediction, thus constraining the output to physically possible solutions (i.e., it is physically impossible to have a state which is less degraded than previous states except for the case of direct service intervention for repair and maintenance, which is out of scope in this work). Another objective is to evaluate whether severity grouping is a viable strategy. Indeed, grouping the LEE stages by type (see Table 1) could be beneficial as a coarse predictor, if it proves to be sufficiently accurate. Thus, in experiment 2.1, all 10 degradation levels are used as labels, while in experiment 2.2 the levels are grouped by type. Three full NHCPP degradation paths (new sample every 6 days, over a 20 year period) with 1200 simulations each are separated into training and validation sets in a $80 / 20$ split. A full degradation path, with 1200 data points, is reserved as the test dataset for final evaluation.

The goal of the third set is to evaluate how the model would perform in a realistic continuous monitoring situation. Here, the dataset is also made of full NHCPP simulations, but in this case, all possible stochasticity is turned on. Again, we assume that we have access to the previous degradation states. We also evaluate class grouping, separating the experiment into two. Three full NHCPP degradation paths (new sample every 6 days, over a 20 year period), each consisting of 1200 MTS data points, are divided into training and validation sets based on a $80 / 20$ split. The test dataset is comprised of one full degradation path, also with 1200 data points unused in training or validation.

Table 5 summarizes the differences between the datasets of the three sets of experiments, including the partition of data between the different subsets.

Table 5: Dataset properties of the different diagnostic experiments.

\begin{tabular}{lcccccc}
\hline \multirow{2}{*}{ Parameter } & \multicolumn{7}{c}{ Experiments } \\
& 1.1 & 1.2 & 2.1 & 2.2 & 3.1 & 3.2 \\
\hline NHCPP severities & $0-9$ & $0,1,6,9$ & $0-9$ & $0-9$ & $0-9$ & $0-9$ \\
Severity type grouping & - & - & - & 0, A, B, C & - & 0, A, B, C \\
Stochastic degradation & - & - & $\checkmark$ & $\checkmark$ & $\checkmark$ & $\checkmark$ \\
Inflow turbulence & $\checkmark$ & $\checkmark$ & $\checkmark$ & $\checkmark$ & $\checkmark$ & $\checkmark$ \\
Aerodynamic uncertainty & - & - & - & - & $\checkmark$ & $\checkmark$ \\
Weather variability & - & - & - & - & $\checkmark$ & $\checkmark$ \\
Num. training samples & 3360 & 3360 & 2880 & 2880 & 2880 & 2880 \\
Num. validation samples & 960 & 960 & 720 & 720 & 720 & 720 \\
Num. testing samples & 480 & 480 & 1200 & 1200 & 1200 & 1200 \\
\hline
\end{tabular}




\subsection{Transformer Architecture}

Here, we introduce the architecture of our Transformer neural network, used to classify long sequence multivariate time-series data and infer the degradation status of the leading edge.

Transformers are models that rely on self-attention mechanisms to highlight and learn dependencies within sequences. Typically, self-attention is implemented by parsing the input sequence into into key $(\mathrm{K})$, query $(\mathrm{Q})$, and value $(\mathrm{V})$ vectors. The attention weights on the values are then obtained by taking the scaled dot products of the query with all keys, and then by applying a softmax function. Using the classical notation [41], this writes:

$$
\operatorname{Attention}(Q, K, V)=\operatorname{softmax}\left(\frac{Q K^{T}}{\sqrt{d_{k}}}\right) V
$$

where $d_{k}$ is the dimension of the key and query vectors and is used to scale the dot product. In practice, $h$ scaled dot-products are used in parallel, within Multi-Head attention layers. A Multi-Head model is able to attend jointly to information gathered from different parts of the input, providing an improvement in representation over a single attention mechanism. The final attention vector is obtained by concatenating each of the dot-product results:

$$
\operatorname{MultiHead}(Q, K, V)=\operatorname{Concat}\left(\text { head }_{1}, \ldots, \text { head }_{h}\right) W^{O}
$$

with

$$
\text { head }_{i}=\operatorname{Attention}\left(Q W_{i}^{Q}, K W_{i}^{K}, V W_{i}^{V}\right)
$$

where the projections are parameter matrices $W_{i}^{Q} \in \mathbb{R}^{d_{\text {model }} \times d_{k}}, W_{i}^{K} \in \mathbb{R}^{d_{\text {model }} \times d_{k}}, V_{i} \in$ $\mathbb{R}^{d_{\text {model }} \times d_{v}}$ and $W^{O} \in \mathbb{R}^{h \cdot d_{v} \times d_{\text {model }}}$.

Multi-Head attention layers are combined with fully connected networks and layer normalization to form the base architecture of each layer of the Transformer stack. We use a novel time-windowing Transformer model which is based on the patch system used in the Vision Transformer (ViT) model [42]. This architecture, as shown in Figure 21, aims to address the quadratic attention bottleneck, an obstacle which limits the length of sequences a Transformer can use. Indeed, the computational complexity of the selfattention layers in Transformer models scales as $\mathcal{O}\left(L^{2}\right)$, where $L$ is length of the input sequence. Given our requirements, the input time-series have a length of $L=60^{\prime} 000$, which is prohibitively resource intensive for standard Transformer models. To alleviate this issue, we propose to divide the data into $\mathrm{N}$ windows along the time-dimension, before passing each window through the learnable input embedding. This allows us to use the full input time-series without resorting to downsampling which would entail losing information.

Before dividing the MTS into windows, each of the individual channels/variables is normalized in time. Then, the input embedding encodes each time-series window into a vector of size $d_{\text {model }}$, which is the latent size used throughout the Transformer. The type of input embedding used has a considerable impact on performance and many embedding types can be envisaged. We choose to use a learnable linear embedding for simplicity, based on initial testing and the literature [42]. A class token is concatenated to the embedded sequence, such that its state at the output of the Transformer is used to infer the degradation severity. Class tokens are commonly used in natural language processing tasks [83], and are an effective way to retrieve categorical information from Transformers. Traditionally, positional encodings are then added to the sequence in order to instil directional information into the model. Contrary to standard NLP Transformer models, we do not use a positional encoding. Two positional encoding methods were 
tested (classical sine functions and simple linear encoding), however, both of these methods hindered classification performance.

The classification head consists of a single hidden layer Multilayer Perceptron (MLP) that processes the class token at the output of the Transformer. It outputs a vector with a size equal to the number of possible degradation classes, which, when passed through a softmax activation, gives the likelihood scores of each class. For each class output $x_{i}$, the softmax likelihood writes:

$$
\operatorname{softmax}\left(x_{i}\right)=\frac{\exp \left(x_{i}\right)}{\left.\sum_{j} \exp \left(x_{j}\right)\right)}
$$

If we aim to predict the degradation at multiple zone along the blade, we can simply stack multiple MLPs, one for each zone.Table 6 summarizes the values used in the Transformer architecture.

Table 6: Parameters of the Transformer architecture.

\begin{tabular}{lc}
\hline Number of windows, $n$ & 300 \\
Window size, $w$ & 200 \\
Internal Transformer dim., $d_{\text {model }}$ & 256 \\
Transformer stack size, $s$ & 6 \\
Num. self-attention heads, $h$ & 8 \\
Self-attention head dim., $d_{\text {head }}$ & 64 \\
Output MLP dim., $d_{M L P}$ & 2048 \\
\hline
\end{tabular}

\subsection{Loss functions}

We first train and evaluate the Transformer model on the datasets with equal amounts of degradation classes, in the sense of a traditional classification problem. Thus, the objective function for this first task is the standard cross-entropy loss:

$$
\mathcal{L}_{C E}(\boldsymbol{y}, \hat{\mathbf{y}})=-\sum_{i=1}^{\mathrm{n}} y_{i} \log \left(\hat{y}_{i}\right)
$$

where $y$ is a one-hot label vector indicating the correct class, $\hat{y}$ is a vector containing the predicted softmax probabilities for each class and $\mathrm{n}$ is the number of classes.

In the second and third set of experiments, it is assumed that the state of the system is known for the previous sampling period. We use this information to enforce physicality; we add a second term to the loss function that penalizes a predicted degradation class that is lower than the known previous class. This second term is the margin ranking loss:

$$
\mathcal{L}_{M R}\left(\hat{\mathrm{c}}, \mathrm{c}_{\text {prev }}\right)=-\max \left(0, \hat{\mathrm{c}}-\mathrm{c}_{\text {prev }}+\mathrm{m}\right)
$$

where $\hat{c}$ is the predicted class, $\mathrm{c}_{\mathrm{prev}}$ is the degradation class of the previous known state, and $\mathrm{m}$ is the margin hyperparameter. Overall, the objective function for the second and third experiments is therefore:

$$
\mathcal{L}_{\text {tot }}=\mathcal{L}_{C E}+\alpha \mathcal{L}_{M R}
$$

where $\alpha$ is a hyperparameter used to balance the two components. 


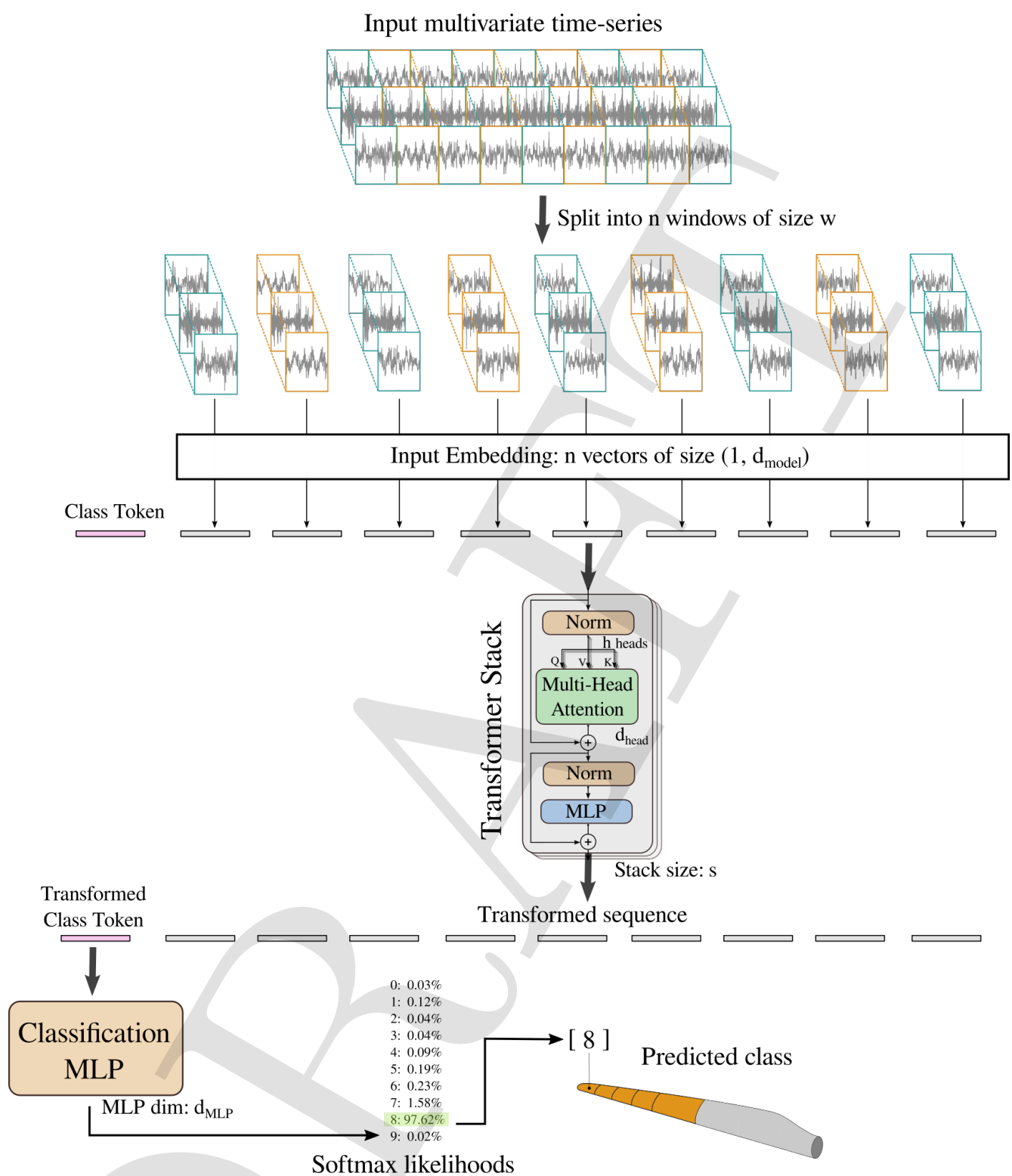

Figure 21. Time-windowing architecture of the novel Transformer model. The input multivariate time-series is split along the time dimension into windows which are then individually passed through the learnable linear embedding. The learnable class token that is added to the sequence is used in the classification MLP head to predict the level of degradation.

\subsection{Training regime}

During training, we use a batch size of 20 along with the Adam optimizer [84] with parameters $\beta_{1}=0.9, \beta_{2}=0.999, \epsilon=10^{-8}$ and a learning rate of $l r=5 \cdot 10^{-5}$ to perform stochastic gradient descent. After 40 epochs of training, the learning rate decays by a factor of 0.2 .

To ensure that the trained model is able to generalize well, and does not overfit, dropout with a 0.3 rate is used in the attention, fully-connected and embedding layers. Furthermore, training and validation metrics (loss and accuracy) are monitored throughout the training sequence, allowing us to halt training without overfitting. Figure 22 shows the training curves of experiment 1.1, highlighting that training for around 60 epochs is sufficient in this case. 


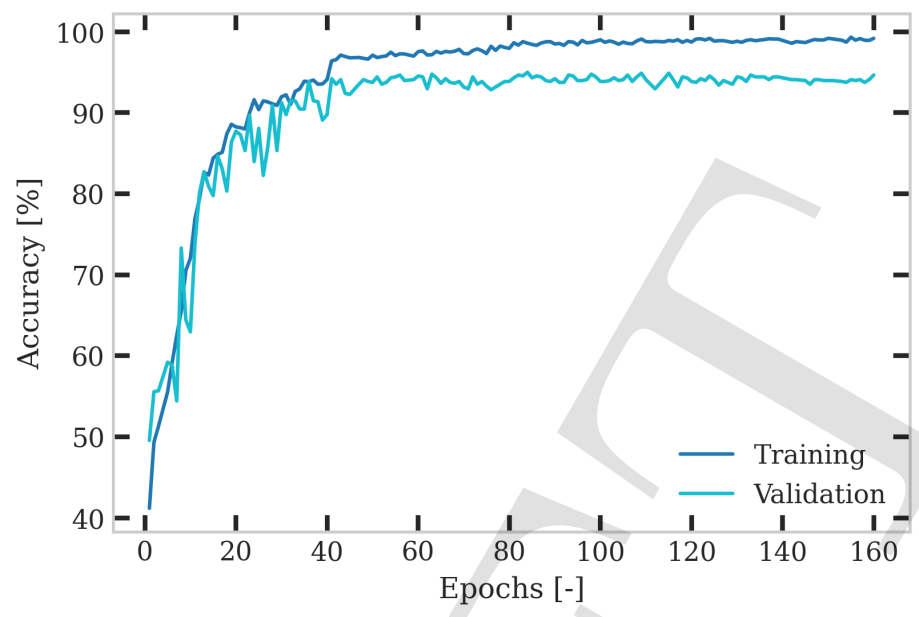

Figure 22. Training and validation accuracy for experiment 1.1 Beyond the 60th epoch no real gain in validation accuracy is made while training accuracy trends to $100 \%$, which indicates overfitting.

\section{Results}

For each diagnostic experiment, a Transformer model is trained on the training dataset, monitored on the validation dataset and final evaluation takes place on the test dataset. All model hyperparameters are static throughout the experimetnts, only the number of training epochs varies, as we choose the epoch with the best validation performance. We report in the following sections the results for all three sets of experiments gathered on the previously un-seen test dataset.

\subsection{Experiment set 1}

The first set of experiments aims to assess the potential of the Transformer for diagnostics given ideal conditions: a balanced data set where each individual degradation class appears in equal measure. Table 7 reports the testing accuracy scores gathered for experiments 1.1 and 1.2. We note a significant difference between the results of the two experiments, where the 4 class setup outperforms the 10 class setup by $30 \%$ in terms of test accuracy. Knowing that Transformers are data-intensive models that respond well to large datasets, this discrepancy can be partly explained by accounting for the number of simulations per individual class. Indeed, as both training sets have the same total number of samples, there are 2.5 times the amount of samples per severity class in experiment 2.2. To factor out the disparity in the amount of data per class, we generate a large version of training dataset of task 1.1, with a total of 10176 training samples and 2544 validation samples. In this experiment (1.1 Large), the number of training samples per class is larger than for experiment 1.2 (1017 versus 840$)$, yet the four class model still outperforms the 10 class model by over $16 \%$. In general it is advantageous to train on larger amounts of severity classes from a risk management perspective, as it allows for more optimal maintenance intervention schemes. However, as highlighted above, a fine-grained approach requires more training data in order to diagnose accurately with high confidence and could result in higher amounts of false positives, which could then lead to an increase in Operation and Maintenance $(O \& M)$ costs. Thus, there is a trade-off between risk minimization via fine-grained diagnostics of LEE severity and increased costs due to higher amounts of false positives and requirements for larger training datasets, which can be optimized to meet risk management policies and budgetary specifications. 
Table 7: Testing classification accuracy of experiments 1.1 and 1.2.

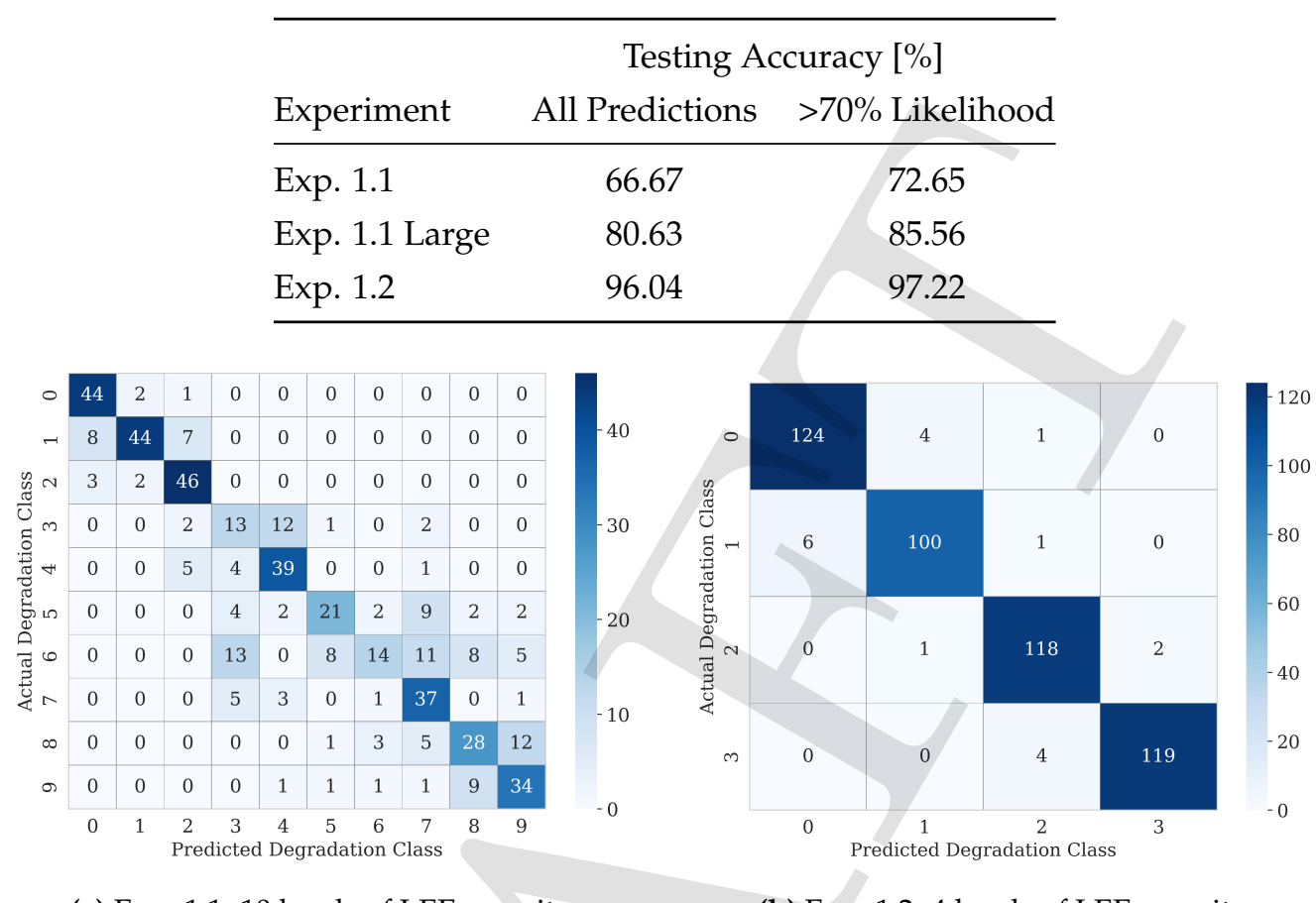

(a) Exp. 1.1: 10 levels of LEE severity.

(b) Exp. 1.2: 4 levels of LEE severity.

Figure 23. Confusion matrices of the predicted severity classes, evaluated on the test datasets of the first set of experiments.

The difference in accuracy can also be explained by the separability of the individual severity classes. We show in Figure 23 the confusion matrices generated on the test sets for experiments 1.1. and 1.2. It can be noted that the Transformer model is able to successfully differentiate between the lowest and highest of erosion severities, as demonstrated by the lack of misclassified samples with large differences in severity. Similar LEE erosion classes exhibit higher amounts of wrongly classified samples. This aligns with our expectations: small increases in the level of erosion only marginally increases the roughness of the leading edge, which has a minor impact on blade aerodynamics. It is therefore more challenging to distinguish between similar LEE severities based on integrated pressure quantities.

\subsection{Experiment set 2}

Here, we assess the use of stochastic degradation paths as diagnostic training points. Table 8 shows accuracy scores gathered on an un-seen degradation path for experiments 2.1 and 2.2. Figure 24 plots the predicted states of each MTS data point alongside the true degradation path, for the test dataset which contains a full NHCPP path with up to 10 possible LE severity classes. In addition, we show the prediction confidence of each model output based on its softmax likelihood. Figure 25 displays the median predicted severity using a three month rolling window.

These results show that this approach is viable and that we are able to successfully detect the jumps in degradation severity with some limitations. The main issue with training on stochastic degradation paths is the data imbalance. Indeed, the training dataset contains a majority of points belonging to classes 0,8 and 9, while classes 5, 6 and 7 are underrepresented. This is reflected in the results, where the accuracy is high for class 0 , but low for classes 5 and 6 . Moreover, prediction confidence is in general lower 
Table 8: Testing classification accuracy of experiments 2.1 and 2.2.

\begin{tabular}{lcc}
\hline & \multicolumn{2}{c}{ Testing Accuracy [\%] } \\
Experiment & All Predictions & $>70 \%$ Likelihood \\
\hline Exp. 2.1 & 65.42 & 78.41 \\
Exp. 2.2 & 67.75 & 69.48 \\
\hline
\end{tabular}

for underrepresented classes. A possible solution to address this is to train on grouped severity classes. We evaluate this approach in experiment 2.2 and plot the resulting predictions in Figures 26 and 27. Overall, this approach yields higher prediction accuracy, but it is still limited. Grouping artificially creates a somewhat balanced set and reduces class sparsity, however, it also leads to increased intra-class heterogeneity. As a result, learning to set the boundaries between the classes becomes more difficult and dependant on the number and the separability of the groups. This is reflected in the disparity of the minimum $70 \%$ confidence accuracy results between experiments 2.1 and 2.2. Indeed, the low intra-class heterogeneity of the 10 class approach results in low confidence predictions for ambiguous samples, while the same ambiguous samples are incorrectly classified with high a likelihood in the grouped approach due to the network accounting for high intra-class variance. There is a balance to be found between the accuracy gain and increase in intra-class heterogeneity induced by class grouping, depending on the number of groups. Should grouping be desired, unsupervised clustering approaches should be envisaged, in order to find the optimal number of groups.

In experiments 2.1 and 2.2, non-physical predictions are penalised via the additional loss term (see equation 16). The result of using this extra component is apparent in Figures 24 and 26: there are noticeably fewer misclassified points below the true degradation path than above it ( $11.5 \%$ vs $23.08 \%$ for experiment $2.1,6.67 \%$ vs $25.58 \%$ for experiment 2.2)

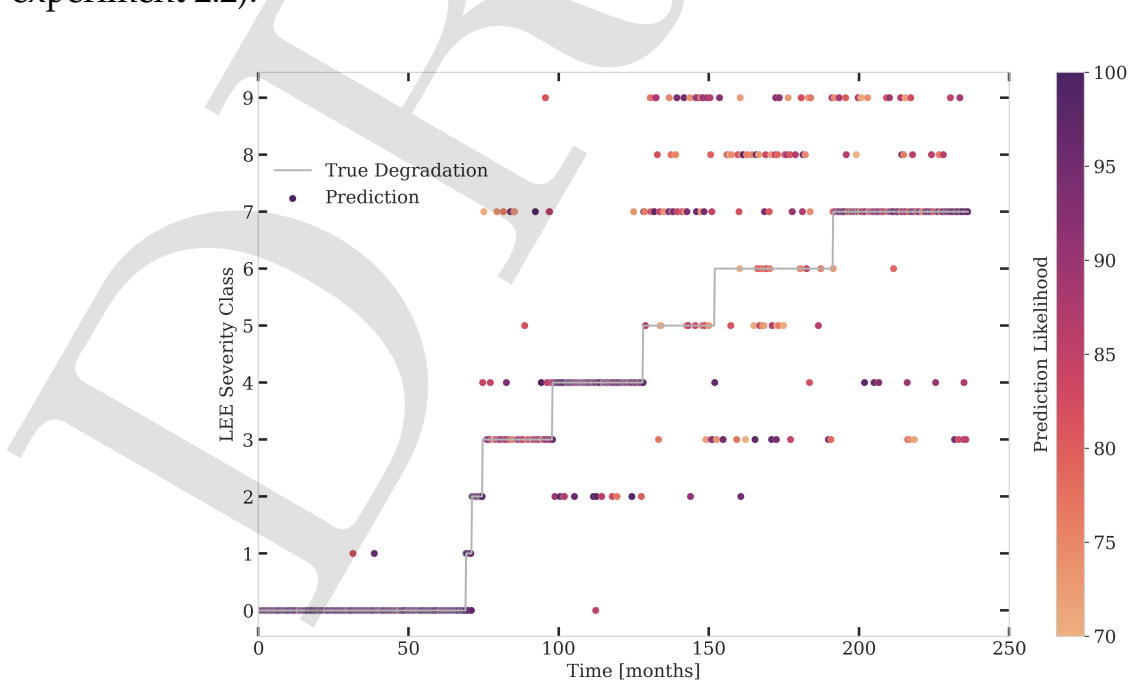

Figure 24. Actual degradation path and predicted erosion severity classes for experiment 2.1. Only predictions with a likelihood greater than $70 \%$ are shown. 


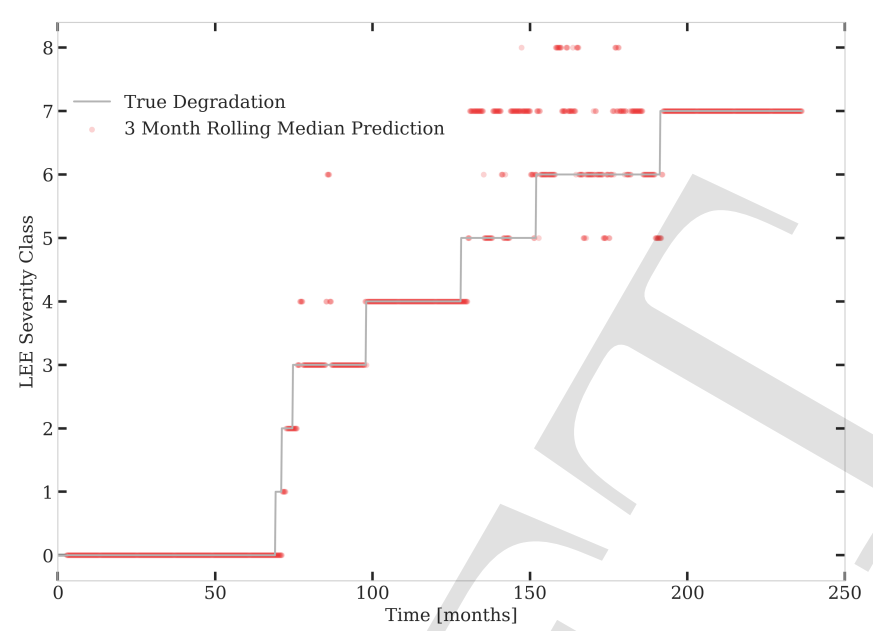

Figure 25. Actual degradation path and median predicted erosion severity classes for experiment 2.1 using a three month rolling window and upper rounding.

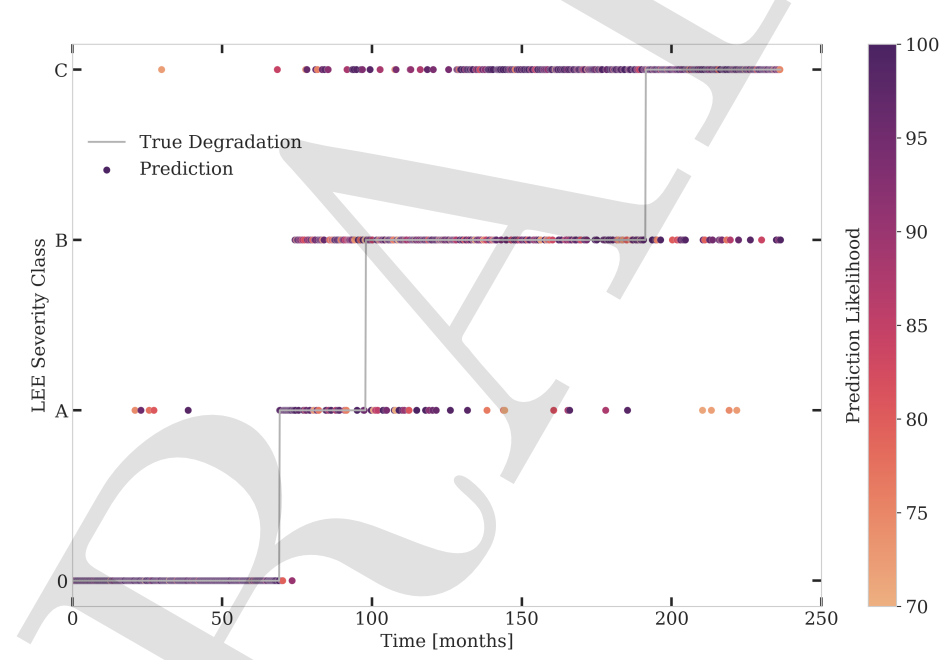

Figure 26. Actual degradation path and predicted erosion severity classes for experiment 2.2. Only predictions with a likelihood greater than $70 \%$ are shown.

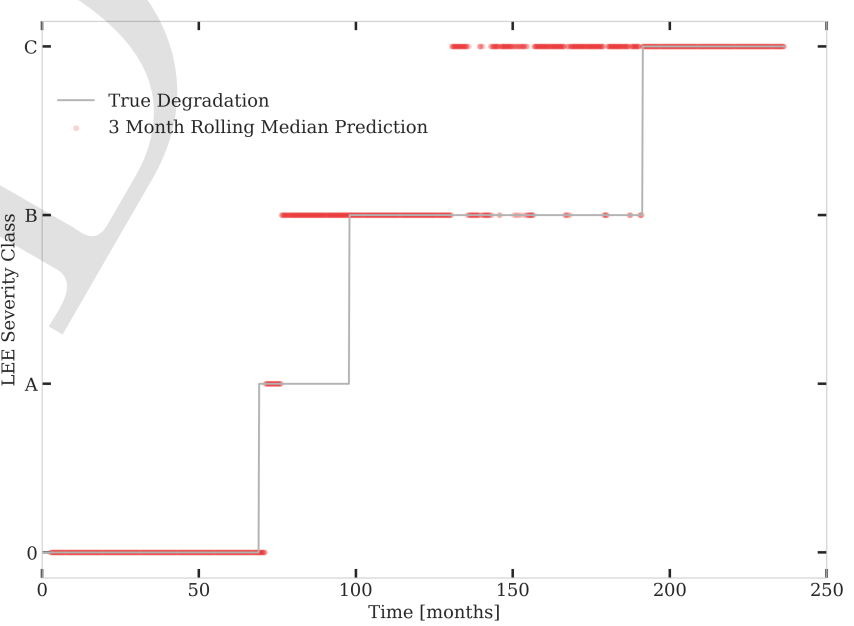

Figure 27. Actual degradation path and median predicted erosion severity classes for experiment 2.2 using a three month rolling window and upper rounding. 


\subsection{Experiment set 3}

In experiments 3.1 and 3.2, we assess how the diagnostics method performs on more uncertain degradation paths, with multiple sources of variability. Not only are there large class imbalances due to the stochastic degradation, but aerodynamic uncertainty (see Figure 7) and long-term weather fluctuations makes it challenging to distinguish between different LEE severities. Weather variability is not commonly used for long-term aero-elastic modelling in the wind energy industry, and is somewhat unrealistic, but the goal is to understand the limitations of the Transformer model by making inference extremely challenging. We report in Table 9 the accuracy scores gathered on an un-seen degradation path for experiments 3.1 and 3.2. Overall, classification accuracy is much lower than previous experiments, but using only high confidence predictions yields a larger boost in accuracy. This can be explained by the inherent stochasticity and the high intra-class variance of this dataset which makes high likelihood predictions rarer.

Table 9: Testing classification accuracy of experiments 3.1 and 3.2.

\begin{tabular}{lcc}
\hline & \multicolumn{2}{c}{ Testing Accuracy [\%] } \\
Experiment & All Predictions & $>70 \%$ Likelihood \\
\hline Exp. 3.1 & 35.00 & 45.75 \\
Exp. 3.2 & 54.67 & 65.22 \\
\hline
\end{tabular}

Figures 28 and 29 show how challenging this final task is, where we see many non-physical misclassified points and an overall low prediction confidence. Here, the data imbalance has a big impact on classification performance. As the training set does not contain any samples with a LEE severity class 9, the network is unable to predict this severity on the given test set. Given these harsh training conditions, it is unsurprising that performance is underwhelming. To mitigate this, class grouping by LEE type is again tested in experiment 3.2 (see Figures 30 and 31). Compared to experiment set 2 , the benefit of grouping classes is clear here: performance is improved by almost $20 \%$. This indicates that class grouping is a viable approach if intra-class heterogeneity is high by default and some classes are completely underrepresented. Nevertheless, even the results for grouped classes are lackluster, indicating that other strategies should be examined to deal with the very imbalanced datasets induced by the stochastic degradation paths. For instance, one could consider conditional retraining methods. By modifying the architecture so that it outputs confidence metrics based on how similar a sample is to the training set, as implemented in [85], one could then launch re-training procedures if multiple test samples that are very dissimilar to the training space are encountered. 


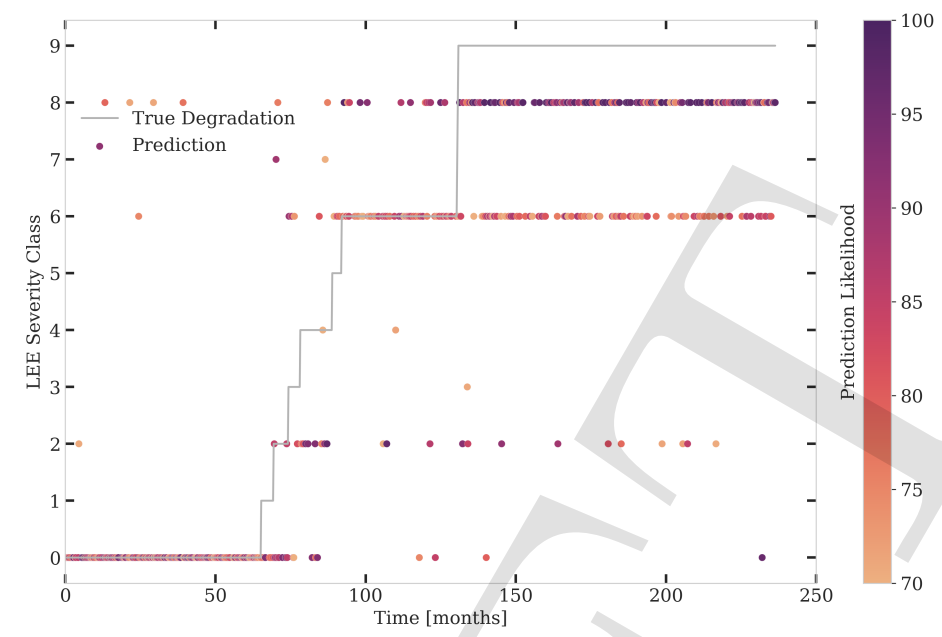

Figure 28. Actual degradation path and predicted erosion severity classes for experiment 3.1.

Only predictions with a likelihood greater than $70 \%$ are shown.

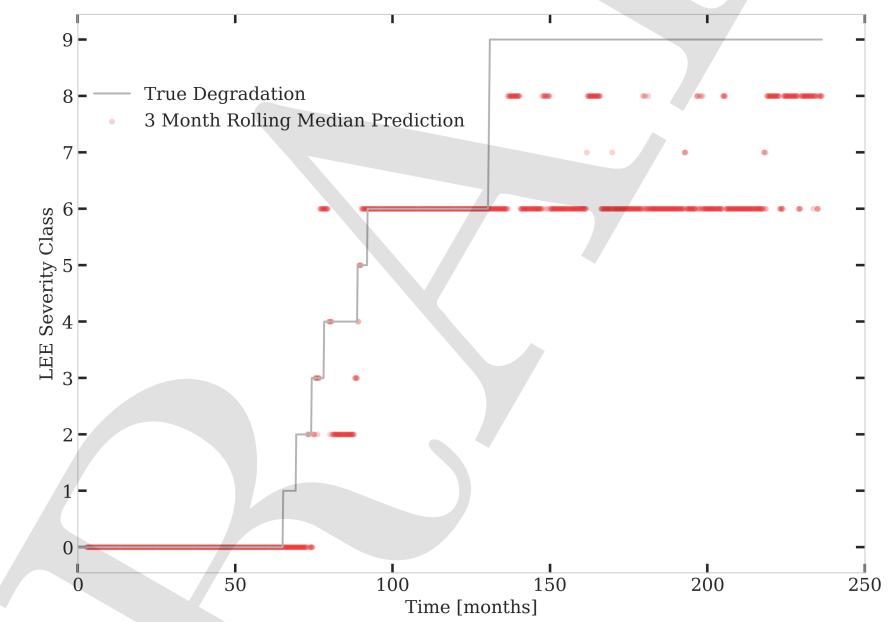

Figure 29. Actual degradation path and median predicted erosion severity classes for experiment 3.1 using a three month rolling window and upper rounding.

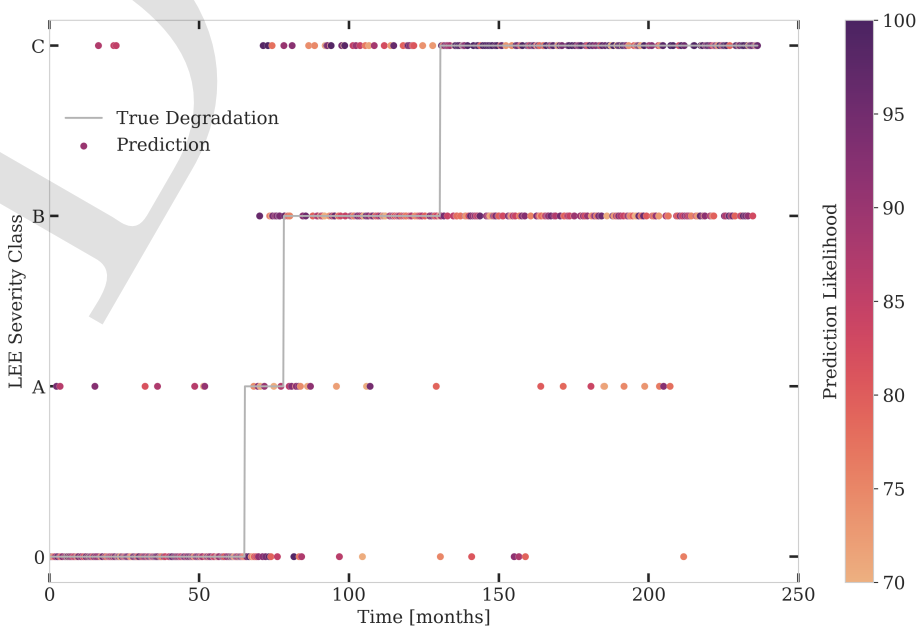

Figure 30. Actual degradation path and predicted erosion severity classes for experiment 3.2. Only predictions with a likelihood greater than $70 \%$ are shown. 


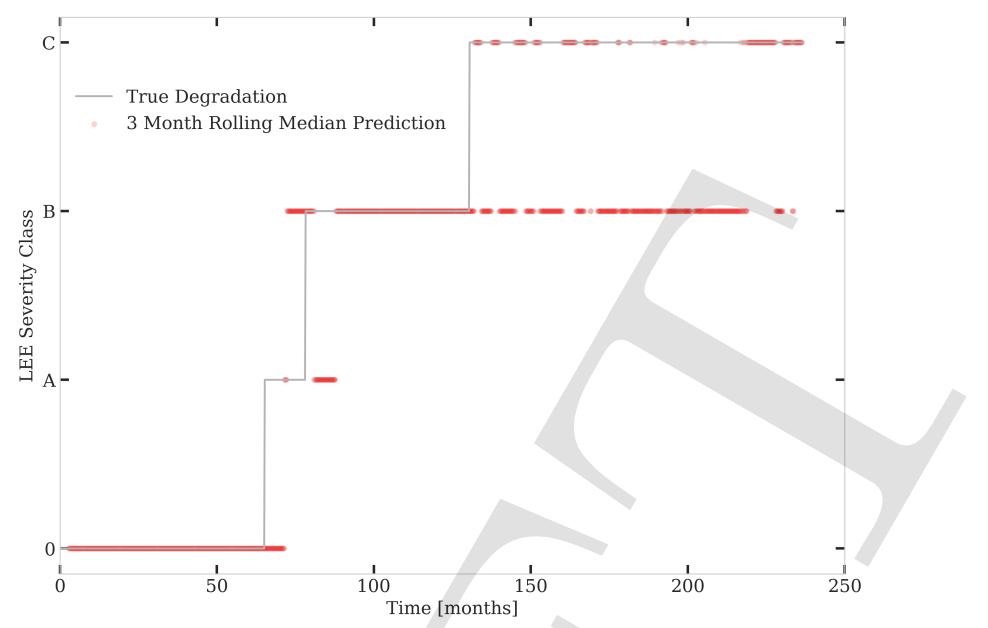

Figure 31. Actual degradation path and median predicted erosion severity classes for experiment 3.2 using a three month rolling window and upper rounding.

Table 11: Transferability and curriculum learning results, for high confidence predictions.

\begin{tabular}{lccc}
\hline & \multicolumn{3}{c}{$>$ 70\% Likelihood Test Accuracy [\%] } \\
Training Set(s) & Set 1.1 & Set 2.1 & Set 3.1 \\
\hline Set 1.1 & - & 80.21 & 30.19 \\
Set 2.1 & 77.11 & - & 32.86 \\
Set 3.1 & 35.58 & 40.97 & - \\
Set 1.1, then 2.1 & 81.35 & 87.29 & 20.52 \\
Set 1.1, then 2.1 \& 3.1 & 84.42 & 88.53 & 50.22 \\
\hline
\end{tabular}

Here, we evaluate the transferability of the different experiments, and assess the use of curriculum learning [86] as a method to improve accuracy on difficult results. In a curriculum learning training setup, the network is progressively exposed to harder datasets. In our implementation, we first train the model on the dataset from experiment 1.1, then progressively add the training data from experiments 2.1 and 3.1. The benefits of this approach should be twofold: (1) the data-intensive Transformer model is exposed to a larger dataset, and (2) the weights of the model are fine-tuned on more challenging samples, which helps to avoid local minima during gradient descent. Tables 10 and 11 show the results of the different transferability and curriculum learning experiments.

Table 10: Transferability and curriculum learning results.

\begin{tabular}{llccc}
\hline & \multicolumn{3}{c}{ Test Accuracy [\%] } \\
Training Set(s) & Set 1.1 & Set 2.1 & Set 3.1 \\
\hline Set 1.1 & - & 71.25 & 28.25 \\
Set 2.1 & 65.00 & - & 29.42 \\
Set 3.1 & 30.00 & 39.25 & - \\
Set 1.1, then 2.1 & 77.71 & 83.50 & 20.33 \\
Set 1.1, then 2.1 \& 3.1 & 77.08 & 80.50 & 37.08 \\
\hline
\end{tabular}

\subsection{Transferability and Curriculum Learning}


- The high accuracy of transferability tests between experiments 1.1 and 2.1 highlight the similarity of these datasets. Moreover, the balanced dataset of experiment 1.1 improves prediction quality for set 2.1.

- Although both sets 1.1 and 2.1 contain all degradation classes, networks trained on these sets do not transfer well to test set 3.1, undoubtedly due to the uncertainty included in this experiment.

- $\quad$ There is better transferability between sets 2.1 and 3.1, than between sets 1.1 and 3.1. This can be explained by the extra loss component which enforces monotonicity in the second and third sets of experiments.

- The first curriculum learning experiment (set 1.1 then 2.1) yields a large performance boost for test sets 1.1 and 2.1. This is to be expected due to the data-intensive nature of Transformers and the similarity between these sets.

- The first curriculum learning experiment reduces transferability to set 3.1. This is an indication of a loss of capacity to generalize to ambiguous data.

- The best test results on set 3.1 are obtained in the second curriculum learning experiment. This suggests that pretraining on sets with reduced stochasticity followed by fine-tuning on uncertain data is an effective approach.

- The second curriculum learning experiment yields the best high confidence accuracy for sets 1.1 and 2.1. Thus, adding difficult, stochastic data points of experiment 3.1 to the training dataset helps with regularization, enabling the model to construct a better internal representation of each severity class.

The curriculum learning test which includes the data from all experiments, leads to the highest high-confidence accuracy for the 2.1 test set. As this set is representative of a degradation path with standard aero-elastic simulation practices, we plot the results in Figures 32 and 33.

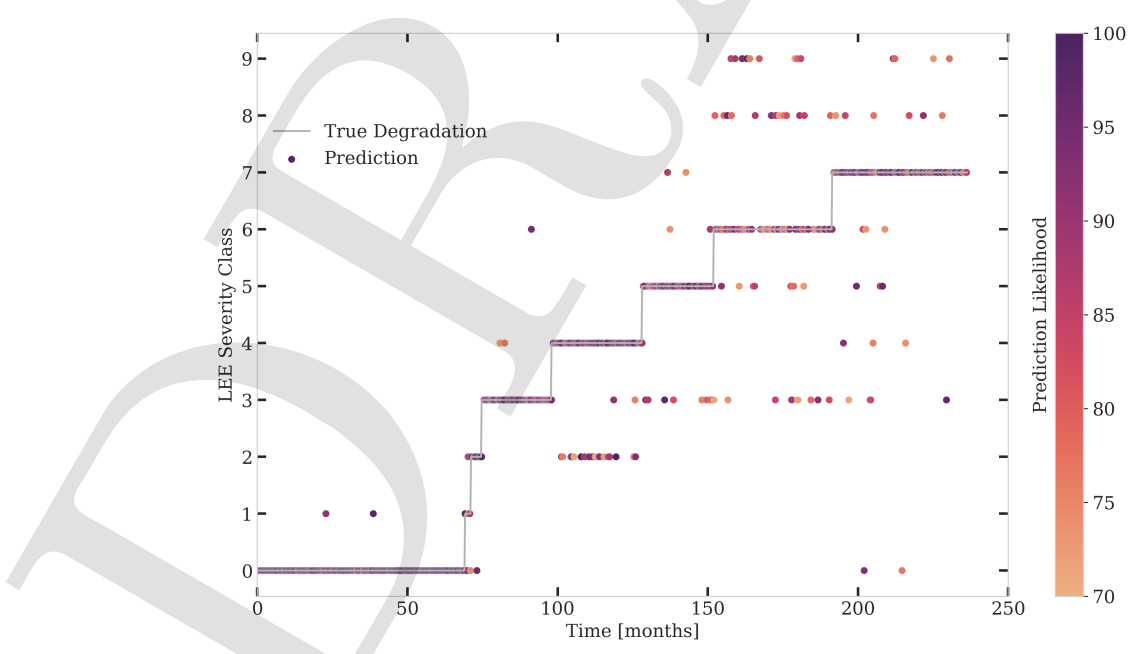

Figure 32. Results for test dataset 2.1 showing actual degradation path and predicted erosion severity classes for the curriculum learning experiment combining all datasets. Only predictions with a likelihood greater than $70 \%$ are shown. 


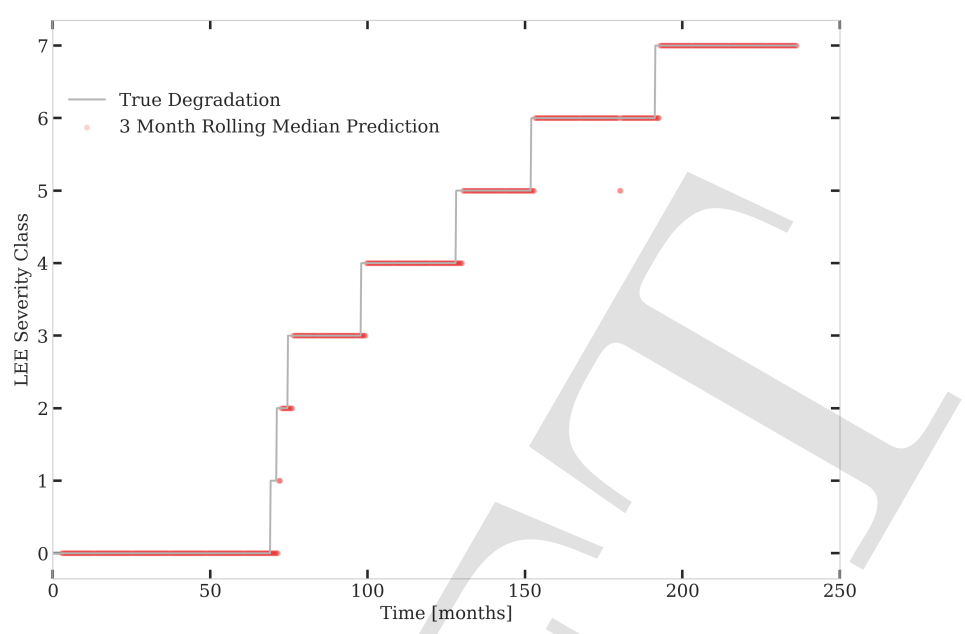

Figure 33. Results for test dataset 2.1 showing actual degradation path and median predicted erosion severity classes for the curriculum learning experiment combining all datasets, using a three month rolling window and upper rounding.

\section{Limitations and Discussion}

All assumptions and modelling choices, constants, choice of probability distributions and their parameters in the proposed NHCPP model for leading edge erosion degradation could in principle be calibrated $[87,88]$. This, however, will prove very challenging given the data-scarce nature of measurements of erosion processes on operating wind turbines in the field over long time horizons. In addition, the impact of field data quantity and quality on model calibration and its implication on the generated NHCPP will need to be closely evaluated. One interesting extension to our NHCPP macro degradation model, is to further couple it to a micro model that describes the relative velocity between particles (including their density and size) and the blade, which leads to an expression for the mass flow of particles impinging the blade leading edge per unit of area [10]. Subsequently, a matricial expression for the number of particles impacting a unit surface is derived to describe the dependency of erosion severity from the atmospheric conditions at the site and the operational settings of the wind turbine.

Similar to the NHCPP, the Gamma process has also been traditionally used for modeling deterioration. A Gamma process has independent non-negative increments, but the shocks follow a Gamma distribution. While in a Poisson process there is a finite number of jumps in a finite time interval, in a Gamma process there are infinite numbers of jumps in a finite time interval. For this reason, the former are suitable for modelling usage such as damage due to sporadic shocks, whereas the Gamma processes are suitable for describing continuous ('graceful' - a succession of tiny increments), monotonic and gradual deterioration. The Gamma process can be regarded as a compound Poisson process of Gamma-distributed increments in which the Poisson rate tends to infinity and increment sizes tend to zero in proportion [89].

One of the main outcomes of the diagnostic experiments is the fact that the Transformer model is data-intensive. Indeed, our results point to the fact that more data almost always aids in classification performance. Furthermore, training on balanced datasets is always desirable, but obtaining these is difficult in real-life monitoring scenarios as it would require gathering data from many systems undergoing different degradation paths. To overcome this difficulty, the coupled NHCPP aeroleastic simulation setup can be used to replicate realistic degradation and produce balanced training sets which 
can then be supplemented with real field data, in a similar manner to the curriculum learning experiments.

As mentioned in the literature review, feature-extraction learning methods are often preferred for MTS classification tasks owing to their higher precision. In this context, We tested the use of the state-of-the-art MINIROCKET method [29] with 10k kernels, combined with a standard linear classifier at the output for our diagnostics problem. This yielded an accuracy of only $55.21 \%$ on the test set of experiment 1.1 and $68.96 \%$ on the test set of experiment 1.2. This highlights the fact that very long sequences are challenging to deal with, while many methods found in the literature are not optimized for such tasks. Furthermore, feature extraction methods may encounter difficulty finding optimal features, due to the fact that this problem features a very slow degradation rate with no flagrant traits from one stochastic sample to another. On the contrary, Transformers are well suited for this kind of input, as the self-attention mechanisms are effective at filtering out unnecessary data while focusing on the parts of the signal that are important.

While the loss term added to penalize non-monotonicity of the degradation process does help to reduce non-physical predictions, it is not strictly enforced. Another option would be to enforce this condition in the softmax output layer. Although this method would most certainly improve accuracy, it would lead to error accumulation when implemented in the context of a continuous monitoring setup. With our approach erroneous predictions can be ignored by using a rolling window for instance. The proposed model could, however, be further improved by additional tuning of the objective function. One such improvement involves incorporation of a inflow-dependant multiplier that reduces the importance of samples that have highly unlikely inflow conditions. Furthermore, while the leading edge degradation process is inherently timedependant, time was not factored into the final diagnostics approach. Initial tests which included a global time stamp in the model input, showed the Transformer overfitting to this variable. A probabilistic loss term that penalizes unlikely severities given the amount of operational time, could be a potential solution to this.

Another possible improvement could be the modification of the training procedure to a bi-directional procedure, similar to the BERT model [83]. In this approach, a pairwise input would be fed into the Transformer, and during the masked pre-training phase, the model would try to predict the class of either the first or second MTS at random. This could lead to a better representation of the data and allow the use of known reference states for comparative diagnostics.

Our proposed approach does not take positional information into account, yet, as shown in Figure 20, this may allow to more clearly distinguish the LEE severity classes along the span of the blade. Inserting positional information into our Transformer model can be achieved in different ways; one possible approach is to encode a graph structure in the Transformer architecture, as implemented in [90].

\section{Conclusion}

In this work we tackle the problem of leading edge erosion of wind turbine blades on a two-fold front. Firstly, we deal with the development of an appropriate model for simulation of such degradation processes and, secondly, we propose a monitoringdriven method for diagnostics of such damage processes. On the first front, we propose a stochastic spatio-temporal empirical model for modelling leading edge erosion degradation based on Non-Homogeneous Compound Poisson Process and couple this to the non-linear time marching OpenFast aeroelastic wind turbine computer simulator. The 
coupled model is able to compute the aeroelastic non-stationary dynamic response of a wind turbine reflecting its behaviour under the effect of leading edge erosion, varying inflow conditions and aerodynamic uncertainties over a long period of degradation time horizon. On the diagnostic front, we adapt a deep neural network, namely a Transformer, to allow for use on very long sequence multivariate time-series. This allows us to solve the problem of spatio-temporal diagnostics of leading edge erosion on wind turbine blades, using the data emanating from the Non-Homogeneous Compound Poisson Process setup; a scheme that intends to emulate data recorded by aeroacoustic sensors placed on wind turbine blades. We showed that the diagnostics model effectively captures the temporal trends induced by long-term degradation of the leading edge. An attractive feature of this method is that it is well-suited for spatio-temporal degradation problems with a very long time horizon.

\section{Acknowledgments}

This work is funded by the BRIDGE Discovery Program of the Swiss National Science Foundation and Innosuisse, project number 40B2 - 0_187087. The authors also acknowledge the support of the European Research Council via the ERC Starting Grant WINDMIL (ERC-2015-StG \#679843) on the topic of Smart Monitoring, Inspection and Life-Cycle Assessment of Wind Turbines.

\section{References}

1. Bogdanoff, J.L.; Kozin, F. Probabilistic models of cumulative damage; Wiley-Interscience: New York, 1985.

2. Herring, R.; Dyer, K.; Martin, F.; Ward, C. The increasing importance of leading edge erosion and a review of existing protection solutions. Renewable and Sustainable Energy Reviews 2019, $115,109382$.

3. Shihavuddin, A.; Chen, X.; Fedorov, V.; Nymark Christensen, A.; Andre Brogaard Riis, N.; Branner, K.; Bjorholm Dahl, A.; Reinhold Paulsen, R. Wind Turbine Surface Damage Detection by Deep Learning Aided Drone Inspection Analysis. Energies 2019, 12.

4. Gertsen, T. Data-driven leading edge erosion detection for wind turbine blades using SCADA data. Master's thesis, TU Delft, the Netherlands, 2019.

5. Shen, J. Classification of Wind Turbine Blade Performance State Through Statistical Methods. Master's thesis, University of Windsor, Canada, 2019.

6. Sareen, A.; Sapre, C.A.; Selig, M.S. Effects of leading edge erosion on wind turbine blade performance. Wind Energy 2014, 17, 1531-1542.

7. Gaudern, N. A practical study of the aerodynamic impact of wind turbine blade leading edge erosion. Journal of Physics: Conference Series 2014, 524, 012031.

8. Bech, J.I.; Hasager, C.B.; Bak, C. Extending the life of wind turbine blade leading edges by reducing the tip speed during extreme precipitation events. Wind Energy Science 2018, $3,729-748$.

9. Groucott, S.; Pugh, K.; Zekos, I.; M Stack, M. A Study of Raindrop Impacts on a Wind Turbine Material: Velocity and Impact Angle Effects on Erosion MAPS at Various Exposure Times. Lubricants 2021, 9.

10. Prieto, R.; Karlsson, T. A model to estimate the effect of variables causing erosion in wind turbine blades. Wind Energy 2021, 24, 1031-1044.

11. Langel, C.M.; Chow, R.; Hurley, O.F.; Dam, C.C.P.V.; Maniaci, D.C.; Ehrmann, R.S.; White, E.B., Analysis of the Impact of Leading Edge Surface Degradation on Wind Turbine Performance. In 33rd Wind Energy Symposium; 2015.

12. Modeling of Rain Drop Erosion in a Multi-MW Wind Turbine, Vol. 9, Turbo Expo: Power for Land, Sea, and Air, 2015.

13. Zidane, I.F.; Saqr, K.M.; Swadener, G.; Ma, X.; Shehadeh, M.F. On the role of surface roughness in the aerodynamic performance and energy conversion of horizontal wind turbine blades: a review. International Journal of Energy Research 2016, 40, 2054-2077. 
14. Schramm, M.; Rahimi, H.; Stoevesandt, B.; Tangager, K. The Influence of Eroded Blades on Wind Turbine Performance Using Numerical Simulations. Energies 2017, 10.

15. Papi, F.; Cappugi, L.; Perez-Becker, S.; Bianchini, A. Numerical Modeling of the Effects of Leading-Edge Erosion and Trailing-Edge Damage on Wind Turbine Loads and Performance. Journal of Engineering for Gas Turbines and Power 2020, 142.

16. Doagou-Rad, S.; Mishnaevsky Jr, L.; Bech, J.I. Leading edge erosion of wind turbine blades: Multiaxial critical plane fatigue model of coating degradation under random liquid impacts. Wind Energy 2020, 23, 1752-1766.

17. Bortolotti, P.; Canet, H.; Bottasso, C.L.; Loganathan, J. Performance of non-intrusive uncertainty quantification in the aeroservoelastic simulation of wind turbines. Wind Energy Science 2019, 4, 397-406.

18. Dimitrov, N. Risk-based approach for rational categorization of damage observations from wind turbine blade inspections. Journal of Physics: Conference Series 2018, 1037, 042021.

19. Shihavuddin, A.S.M.; Chen, X.; Fedorov, V.; Nymark Christensen, A.; Andre Brogaard Riis, N.; Branner, K.; Bjorholm Dahl, A.; Reinhold Paulsen, R. Wind Turbine Surface Damage Detection by Deep Learning Aided Drone Inspection Analysis. Energies 2019, 12, 676. Number: 4 Publisher: Multidisciplinary Digital Publishing Institute, doi:10.3390/en12040676.

20. Ruiz, A.P.; Flynn, M.; Large, J.; Middlehurst, M.; Bagnall, A. The great multivariate time series classification bake off: a review and experimental evaluation of recent algorithmic advances. Data Mining and Knowledge Discovery 2020. doi:10.1007/s10618-020-00727-3.

21. Wang, Z.; Yan, W.; Oates, T. Time series classification from scratch with deep neural networks: A strong baseline. 2017 International joint conference on neural networks (IJCNN). IEEE, 2017, pp. 1578-1585.

22. Middlehurst, M.; Large, J.; Bagnall, A. The Canonical Interval Forest (CIF) Classifier for Time Series Classification. arXiv:2008.09172 [cs, eess] 2020. arXiv: 2008.09172.

23. Stallone, A.; Cicone, A.; Materassi, M. New insights and best practices for the successful use of Empirical Mode Decomposition, Iterative Filtering and derived algorithms. Scientific Reports 2020, 10.

24. Barbosh, M.; Singh, P.; Sadhu, A. Empirical mode decomposition and its variants: a review with applications in structural health monitoring. Smart Materials and Structures 2020, $29,093001$.

25. Górecki, T.; Łuczak, M. Multivariate time series classification with parametric derivative dynamic time warping. Expert Systems with Applications 2015, 42, 2305 - 2312. doi: https:/ / doi.org/10.1016/j.eswa.2014.11.007.

26. Lin, J.; Khade, R.; Li, Y. Rotation-invariant similarity in time series using bag-of-patterns representation. Journal of Intelligent Information Systems 2012, 39, 287-315.

27. Schäfer, P.; Leser, U. Multivariate Time Series Classification with WEASEL+MUSE. arXiv:1711.11343 [cs] 2018. arXiv: 1711.11343.

28. Dempster, A.; Petitjean, F.; Webb, G.I. ROCKET: exceptionally fast and accurate time series classification using random convolutional kernels. Data Mining and Knowledge Discovery 2020, 34, 1454-1495. doi:10.1007/s10618-020-00701-z.

29. Dempster, A.; Schmidt, D.F.; Webb, G.I. MINIROCKET: A Very Fast (Almost) Deterministic Transform for Time Series Classification. arXiv:2012.08791 [cs, stat] 2021. arXiv: 2012.08791, doi:10.1145/3447548.3467231.

30. Dau, H.A.; Keogh, E.; Kamgar, K.; Yeh, C.C.M.; Zhu, Y.; Gharghabi, S.; Ratanamahatana, C.A.; Yanping.; Hu, B.; Begum, N.; Bagnall, A.; Mueen, A.; Batista, G.; Hexagon-ML. The UCR Time Series Classification Archive, 2018.

31. Yang, L.; Zhang, Z. A Conditional Convolutional Autoencoder Based Method for Monitoring Wind Turbine Blade Breakages. IEEE Transactions on Industrial Informatics 2020.

32. Krizhevsky, A.; Sutskever, I.; Hinton, G.E. ImageNet classification with deep convolutional neural networks. Communications of the ACM 2017, 60, 84-90. doi:10.1145/3065386.

33. Fawaz, H.I.; Forestier, G.; Weber, J.; Idoumghar, L.; Muller, P.A. Deep learning for time series classification: a review. arXiv:1809.04356 [cs, stat] 2019. arXiv: 1809.04356, doi: 10.1007/s10618-019-00619-1.

34. Liu, C.; Hsaio, W.; Tu, Y. Time Series Classification With Multivariate Convolutional Neural Network. IEEE Transactions on Industrial Electronics 2019, 66, 4788-4797. Conference Name: IEEE Transactions on Industrial Electronics, doi:10.1109/TIE.2018.2864702. 
35. Fawaz, H.I.; Forestier, G.; Weber, J.; Idoumghar, L.; Muller, P.A. Evaluating surgical skills from kinematic data using convolutional neural networks. arXiv:1806.02750 [cs] 2018, 11073, 214-221. arXiv: 1806.02750, doi:10.1007/978-3-030-00937-3_25.

36. Fawaz, H.I.; Lucas, B.; Forestier, G.; Pelletier, C.; Schmidt, D.F.; Weber, J.; Webb, G.I.; Idoumghar, L.; Muller, P.A.; Petitjean, F. InceptionTime: Finding AlexNet for Time Series Classification. arXiv:1909.04939 [cs, stat] 2020. arXiv: 1909.04939, doi:10.1007/s10618-02000710-y.

37. Rumelhart, D.E.; Hinton, G.E.; Williams, R.J. Learning representations by back-propagating errors. Nature 1986, 323, 533-536. Number: 6088 Publisher: Nature Publishing Group, doi: 10.1038/323533a0.

38. Hochreiter, S.; Schmidhuber, J. Long short-term memory. Neural computation 1997, 9, 17351780.

39. Lipton, Z.C.; Kale, D.C.; Elkan, C.; Wetzel, R. Learning to Diagnose with LSTM Recurrent Neural Networks. arXiv:1511.03677 [cs] 2017. arXiv: 1511.03677.

40. Karim, F.; Majumdar, S.; Darabi, H.; Harford, S. Multivariate LSTM-FCNs for time series classification. Neural Networks 2019, 116, 237-245.

41. Vaswani, A.; Shazeer, N.; Parmar, N.; Uszkoreit, J.; Jones, L.; Gomez, A.N.; Kaiser, Ł.; Polosukhin, I. Attention is all you need. Advances in neural information processing systems, 2017, pp. 5998-6008.

42. Dosovitskiy, A.; Beyer, L.; Kolesnikov, A.; Weissenborn, D.; Zhai, X.; Unterthiner, T.; Dehghani, M.; Minderer, M.; Heigold, G.; Gelly, S.; Uszkoreit, J.; Houlsby, N. An Image is Worth 16x16 Words: Transformers for Image Recognition at Scale. arXiv:2010.11929 [cs] 2020. arXiv: 2010.11929.

43. Zhou, H.; Zhang, S.; Peng, J.; Zhang, S.; Li, J.; Xiong, H.; Zhang, W. Informer: Beyond Efficient Transformer for Long Sequence Time-Series Forecasting. arXiv:2012.07436 [cs] 2020. arXiv: 2012.07436.

44. Zerveas, G.; Jayaraman, S.; Patel, D.; Bhamidipaty, A.; Eickhoff, C. A Transformer-based Framework for Multivariate Time Series Representation Learning. arXiv:2010.02803 [cs] 2020. arXiv: 2010.02803.

45. Scarselli, F.; Gori, M.; Tsoi, A.C.; Hagenbuchner, M.; Monfardini, G. The Graph Neural Network Model. IEEE Transactions on Neural Networks 2009, 20, 61-80. Conference Name: IEEE Transactions on Neural Networks, doi:10.1109/TNN.2008.2005605.

46. Xu, H.; Duan, Z.; Bai, Y.; Huang, Y.; Ren, A.; Yu, Q.; Zhang, Q.; Wang, Y.; Wang, X.; Sun, Y.; Wang, W. Multivariate Time Series Classification with Hierarchical Variational Graph Pooling. arXiv:2010.05649 [cs] 2020. arXiv: 2010.05649.

47. Yu, C.; Ma, X.; Ren, J.; Zhao, H.; Yi, S. Spatio-Temporal Graph Transformer Networks for Pedestrian Trajectory Prediction, 2020, [arXiv:cs.CV/2005.08514].

48. Li, C.; Zhang, S.; Qin, Y.; Estupinan, E. A systematic review of deep transfer learning for machinery fault diagnosis. Neurocomputing 2020, 407, 121-135. doi: 10.1016/j.neucom.2020.04.045.

49. Long, M.; Cao, Y.; Wang, J.; Jordan, M.I. Learning Transferable Features with Deep Adaptation Networks. arXiv:1502.02791 [cs] 2015. arXiv: 1502.02791.

50. Aghabozorgi, S.; Seyed Shirkhorshidi, A.; Ying Wah, T. Time-series clustering - A decade review. Information Systems 2015, 53, 16-38.

51. Izakian, H.; Pedrycz, W.; Jamal, I. Fuzzy clustering of time series data using dynamic time warping distance. Engineering Applications of Artificial Intelligence 2015, 39, 235-244.

52. Franceschi, J.Y.; Dieuleveut, A.; Jaggi, M. Unsupervised Scalable Representation Learning for Multivariate Time Series. arXiv:1901.10738 [cs, stat] 2020. arXiv: 1901.10738.

53. Chandola, V.; Banerjee, A.; Kumar, V. Anomaly detection: A survey. ACM computing surveys (CSUR) 2009, 41, 1-58.

54. Chan, P.K.; Mahoney, M.V. Modeling multiple time series for anomaly detection. Fifth IEEE International Conference on Data Mining (ICDM'05). IEEE, 2005, pp. 8-pp.

55. Mikalsen, K.Ø.; Bianchi, F.M.; Soguero-Ruiz, C.; Jenssen, R. Time series cluster kernel for learning similarities between multivariate time series with missing data. Pattern Recognition 2018, 76, 569-581.

56. Chalapathy, R.; Chawla, S. Deep Learning for Anomaly Detection: A Survey. arXiv:1901.03407 [cs, stat] 2019. arXiv: 1901.03407.

57. Kim, T.Y.; Cho, S.B. Web traffic anomaly detection using C-LSTM neural networks. Expert Systems with Applications 2018, 106, 66-76. 
58. Zhang, C.; Song, D.; Chen, Y.; Feng, X.; Lumezanu, C.; Cheng, W.; Ni, J.; Zong, B.; Chen, H.; Chawla, N.V. A deep neural network for unsupervised anomaly detection and diagnosis in multivariate time series data. Proceedings of the AAAI Conference on Artificial Intelligence, 2019, Vol. 33, pp. 1409-1416.

59. Goodfellow, I.; Pouget-Abadie, J.; Mirza, M.; Xu, B.; Warde-Farley, D.; Ozair, S.; Courville, A.; Bengio, Y. Generative adversarial nets. Advances in neural information processing systems 2014, 27, 2672-2680.

60. Li, D.; Chen, D.; Jin, B.; Shi, L.; Goh, J.; Ng, S.K. MAD-GAN: Multivariate anomaly detection for time series data with generative adversarial networks. International Conference on Artificial Neural Networks. Springer, 2019, pp. 703-716.

61. Rombach, K.; Michau, G.; Fink, O. Contrastive Learning for Fault Detection and Diagnostics in the Context of Changing Operating Conditions and Novel Fault Types. Sensors 2021, 21.

62. Du, Y.; Zhou, S.; Jing, X.; Peng, Y.; Wu, H.; Kwok, N. Damage detection techniques for wind turbine blades: A review. Mechanical Systems and Signal Processing 2020, 141, 106445.

63. Dervilis, N.; Choi, M.; Taylor, S.; Barthorpe, R.; Park, G.; Farrar, C.; Worden, K. On damage diagnosis for a wind turbine blade using pattern recognition. Journal of sound and vibration 2014, 333, 1833-1850.

64. Lei, J.; Liu, C.; Jiang, D. Fault diagnosis of wind turbine based on Long Short-term memory networks. Renewable energy 2019, 133, 422-432.

65. Yang, L.; Zhang, Z. A Conditional Convolutional Autoencoder Based Method for Monitoring Wind Turbine Blade Breakages. IEEE Transactions on Industrial Informatics 2020.

66. Regan, T.; Beale, C.; Inalpolat, M. Wind Turbine Blade Damage Detection Using Supervised Machine Learning Algorithms. Journal of Vibration and Acoustics 2017, 139. doi: 10.1115/1.4036951.

67. Joshuva, A.; Sugumaran, V. A data driven approach for condition monitoring of wind turbine blade using vibration signals through best-first tree algorithm and functional trees algorithm: A comparative study. ISA transactions 2017, 67, 160-172.

68. Zhang, L.; Liu, K.; Wang, Y.; Omariba, Z.B. Ice Detection Model of Wind Turbine Blades Based on Random Forest Classifier. Energies 2018, 11, 2548. doi:10.3390/en11102548.

69. Weijtjens, W.; Avendaño-Valencia, L.D.; Devriendt, C.; Chatzi, E. Cost-effective vibration based detection of wind turbine blade icing from sensors mounted on the tower. 9th European workshop on structural health monitoring (EWSHM 2018), Manchester, UK, 2018, pp. 10-13.

70. Jiang, J.R.; Lee, J.E.; Zeng, Y.M. Time Series Multiple Channel Convolutional Neural Network with Attention-Based Long Short-Term Memory for Predicting Bearing Remaining Useful Life. Sensors 2020, 20, 166. Number: 1 Publisher: Multidisciplinary Digital Publishing Institute, doi:10.3390/s20010166.

71. Jin, C.c.; Chen, X. An end-to-end framework combining time-frequency expert knowledge and modified transformer networks for vibration signal classification. Expert Systems with Applications 2021, 171, 114570. doi:10.1016/j.eswa.2021.114570.

72. Abdallah, I.; Natarajan, A.; Sørensen, J. Impact of uncertainty in airfoil characteristics on wind turbine extreme loads. Renewable Energy 2015, 75, 283 - 300.

73. Han, W.; Kim, J.; Kim, B. Effects of contamination and erosion at the leading edge of blade tip airfoils on the annual energy production of wind turbines. Renewable Energy 2018, $115,817-823$.

74. Billingsley, P. Probability and Measure, 3rd edition; JOHN WILEY \& SONS: New York, 1995.

75. Sanchez-Silva, M.; Riascos-Ochoa, J. Seismic risk models for aging and deteriorating buildings and civil infrastructure. In Handbook of Seismic Risk Analysis and Management of Civil Infrastructure Systems; Tesfamariam, S.; Goda, K., Eds.; Woodhead Publishing: Cambridge, 2013; chapter 15, pp. 387-409.

76. Taylor, H.M.; Karlin, S. An introduction to stochastic modeling, 3rd edition; Academic Press: San Diego, 1998.

77. Chen, C.; Savits, T. Some remarks on compound nonhomogeneous Poisson processes. Statistics $\mathcal{E}$ Probability Letters 1993, 17, 179 - 187.

78. 61400-1. Wind Turbines, Part 1 Design Requirements. Technical Report IEC 61400-1:2005(E), International Electrotechnical Commission, 2005.

79. Dimitrov, N.; Natarajan, A.; Kelly, M. Model of wind shear conditional on turbulence and its impact on wind turbine loads. Wind Energy 2014, 18, 1917-1931. 
80. Saltelli, A.; Annoni, P.; Azzini, I.; Campolongo, F.; Ratto, M.; Tarantola, S. Variance based sensitivity analysis of model output. Design and estimator for the total sensitivity index. Computer Physics Communications 2010, 181, 259-270.

81. Jonkman, J.; Buhl, M. FAST user's guide. Technical Report NREL/EL-500-38230, National Renewable Energy Laboratory, Golden, CO., USA, 2005.

82. Kaimal, J.C.; Wyngaard, J.C.; Izumi, Y.; Coté, O.R. Spectral characteristics of surface-layer turbulence. Quarterly Journal of the Royal Meteorological Society 1972, 98, 563-589.

83. Devlin, J.; Chang, M.W.; Lee, K.; Toutanova, K. BERT: Pre-training of Deep Bidirectional Transformers for Language Understanding. arXiv:1810.04805 [cs] 2019. arXiv: 1810.04805.

84. Kingma, D.P.; Ba, J. Adam: A Method for Stochastic Optimization. arXiv:1412.6980 [cs] 2014. arXiv: 1412.6980.

85. Avendaño-Valencia, L.D.; Chatzi, E.N. Sensitivity driven robust vibration-based damage diagnosis under uncertainty through hierarchical Bayes time-series representations. Procedia Engineering 2017, 199, 1852-1857.

86. Bengio, Y.; Louradour, J.; Collobert, R.; Weston, J. Curriculum learning. Proceedings of the 26th Annual International Conference on Machine Learning - ICML '09; ACM Press: Montreal, Quebec, Canada, 2009; pp. 1-8. doi:10.1145/1553374.1553380.

87. Guida, M.; Calabria, R.; Pulcini, G. Bayes inference for a non-homogeneous Poisson process with power intensity law (reliability). IEEE Transactions on Reliability 1989, 38, 603-609.

88. Grabski, F. Nonhomogeneous compound Poisson process application to modeling of random processes related to accidents in the Baltic Sea waters and ports. Journal of Polish Safety and Reliability Association 2018, Vol. 9, No. 3, 21-30.

89. Noortwijk, J. A survey of the application of gamma processes in maintenance. Reliability Engineering and Systems Safety 2009, 94, 2-21.

90. Mialon, G.; Chen, D.; Selosse, M.; Mairal, J. GraphiT: Encoding Graph Structure in Transformers. arXiv:2106.05667 [cs] 2021. arXiv: 2106.05667. 


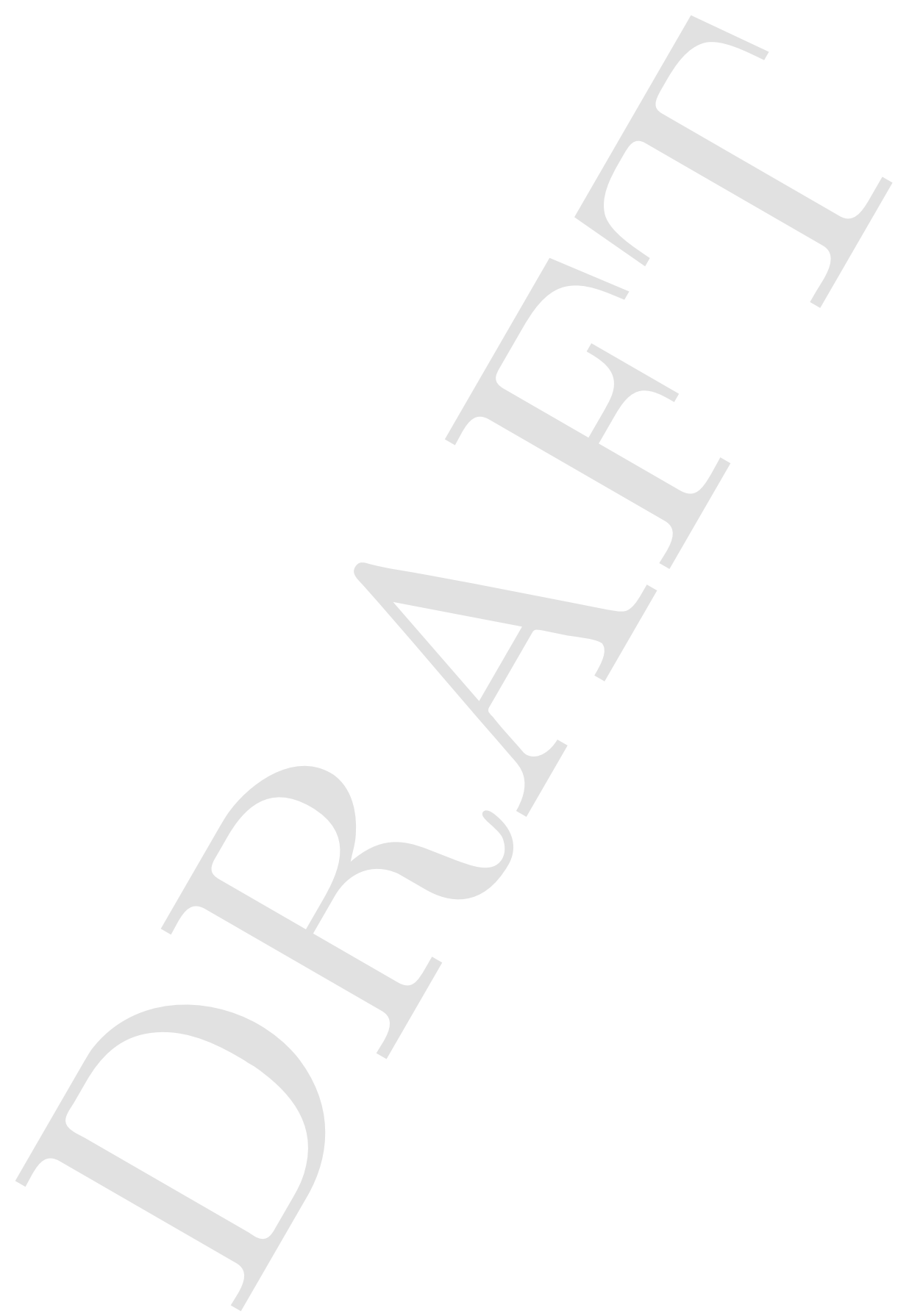




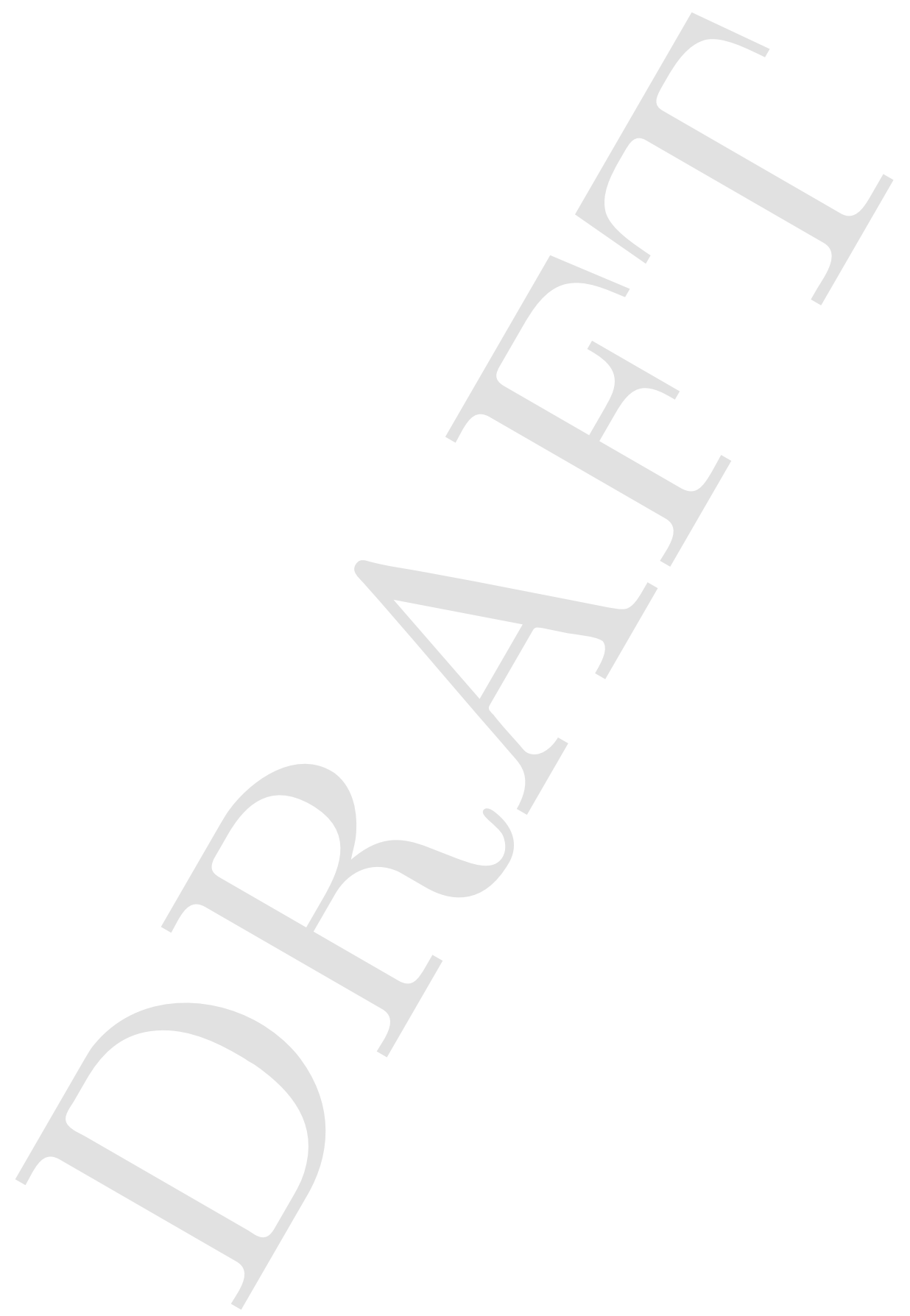

